\title{
Extremal black brane solutions in five-dimensional gauged supergravity
}

\section{Susanne Barisch-Dick, ${ }^{a, b}$ Gabriel Lopes Cardoso, ${ }^{c}$ Michael Haack ${ }^{a}$ and Suresh Nampuri ${ }^{d}$}

${ }^{a}$ Arnold Sommerfeld Center for Theoretical Physics, Ludwig-Maximilians-Universität München, Theresienstrasse 37, 80333 München, Germany

${ }^{b}$ Max-Planck-Institut für Physik, Föhringer Ring 6, 80805 München, Germany

${ }^{c} C A M G S D$, Departamento de Matemática, Instituto Superior Técnico, Universidade Técnica de Lisboa, Av. Rovisco Pais, 1049-001 Lisboa, Portugal

${ }^{d}$ Laboratoire de Physique Théorique, Unité Mixte du CNRS et de l'École Normale Supérieure, École Normale Supérieure,

24 rue Lhomond, 75231 Paris Cedex 05, France

E-mail: susanne.barisch@physik.uni-muenchen.de, gcardoso@math.ist.utl.pt, michael.haack@physik.uni-muenchen.de, nampuri@gmail.com

ABSTRACT: We study stationary black brane solutions in the context of $N=2, \mathrm{U}(1)$ gauged supergravity in five dimensions. Using the formalism of first-order flow equations, we construct examples of extremal black brane solutions that include Nernst branes, i.e. extremal black brane solutions with vanishing entropy density, as well as black branes with cylindrical horizon topology, whose entropy density can be computed from a Cardy formula of the dual CFT.

KeYwords: Black Holes in String Theory, Extended Supersymmetry

ARXIV EPRINT: 1211.0832 


\section{Contents}

1 Introduction 1

2 First-order flow equations for stationary solutions 3

2.1 Flow equations in big moduli space 3

$\begin{array}{ll}2.2 \text { Hamiltonian constraint } & 10\end{array}$

3 Reducing to four dimensions 11

4 Solutions $\quad 12$

$\begin{array}{lll}4.1 & \text { Solutions with constant scalar fields } X^{A} & 12\end{array}$

4.1.1 Taking $P^{A} \neq 0, \hat{q}_{A}=0, \hat{J} \neq 0 \quad 13$

4.1.2 Taking $P^{A}=0, q_{A}=0, \hat{J} \neq 0 \quad 16$

$\begin{array}{lll}4.2 & \text { Solutions with non-constant scalar fields } X^{A} & 18\end{array}$

$\begin{array}{lll}\text { 4.2.1 Solution with a single running scalar } & 19\end{array}$

4.2.2 Solution with two running scalars, $\hat{J}=0 \quad 20$

4.2.3 Solutions with two running scalars, $\hat{J} \neq 0 \quad 21$

$\begin{array}{ll}\text { A Einstein equations } & 24\end{array}$

B Relating five- and four-dimensional flow equations $\quad 24$

C A different first-order rewriting $\quad 30$

\section{Introduction}

Extremal black solutions in low energy effective theories arising from string theories always offer scope for understanding aspects of the Hilbert space of the quantum gravity theory that arises in this context. In the fortuitous cases where the asymptotics of the geometry or the near horizon geometry is either global AdS or a quotient of the Poincare patch of AdS, these solutions may be analyzed in terms of thermal ensembles in the holographic dual CFTs, and hence either offer a bulk view of strongly coupled field theory processes in the boundary theory or a microscopic understanding of the thermodynamic properties of the extremal black solutions [1].

There has been extensive progress in constructing and analyzing extremal black hole solutions from both the bulk and the holographic CFT points of view. Recent developments in the construction of extremal black solutions with non-trivial scalar fields in gauged supergravity theories in four dimensions show that the presence of the fluxes can give rise to a wide variety of asymptotically non-flat backgrounds [2-8]. One of the salient features of the solution space of gauged supergravity actions in four dimensions is the 
existence of horizons with non-spherical horizon topology, such as $\mathbb{R}^{2}$, and a specific subset of these solutions involve extremal black branes with zero area density and hence zero entropy density [6,9-11]. The thermodynamic behavior of these systems are closest to real condensed matter systems (low entropy at zero temperature) and in cases where these brane solutions can be found in asymptotic AdS backgrounds, they can in principle be used to study dual condensed matter systems with quantum phase transitions at zero temperature, as in [12-15]. An example of an extremal black brane solution that satisfies the third law of thermodynamics (the Nernst law) was obtained in [6] as a solution to the STU model of $N=2, \mathrm{U}(1)$ gauged supergravity in four dimensions. However, it was also found to be difficult to obtain analytic solutions describing extremal black brane solutions in asymptotic $A d S_{4}$ backgrounds which, as recalled above, represents a worthy endeavor in view of possible applications in holography. Hence, in the following, we shift focus to gauged supergravity in five dimensions, with the intent of finding extremal solutions in asymptotic $A d S_{5}$ backgrounds, or extremal solutions with a near horizon geometry given in terms of a quotient of the Poincaré patch of $A d S_{3}$, so that one can use the dual CFT to arrive at a microscopic understanding of the black brane entropy density. We will not rely on supersymmetry to construct these extremal solutions. Various types of extremal (not necessarily supersymmetric) five-dimensional black solutions with flat horizons have already been discussed in [7, 12, 15-24].

In this paper, we follow roughly the pattern of exploration set up in $[3,6]$ for the four-dimensional case. We begin by rewriting the five-dimensional $N=2, \mathrm{U}(1)$ gauged supergravity action in terms of squares of first-order flow equations. In the ungauged case, it is known [25] that there exist multiple rewritings based on different 'superpotentials', depending on the charges that are turned on. In the presence of fluxes, we observe a similar feature. The flow equations we obtain are supported by electric charges, magnetic fields and fluxes of electric type. The solutions we construct include Nernst solutions in asymptotic $A d S_{5}$ backgrounds (i.e. black solutions with vanishing entropy density) as well as non-Nernst black brane solutions that describe extremal $\mathrm{BTZ} \times \mathbb{R}^{2}$-solutions. The latter have a cylindrical horizon topology $S^{1} \times \mathbb{R}^{2}$, with the geometry being a quotient of the Poincaré patch of $A d S_{3}$ trivially fibered over an $\mathbb{R}^{2}$ surface. The near-horizon $A d S_{3} \times \mathbb{R}^{2}$ solution has been obtained before in $[18,19]$ using an analysis based on supersymmetry.

We can immediately compute the entropy density of the $\mathrm{BTZ} \times \mathbb{R}^{2}$ black brane by using the Cardy formula of the dual CFT, thereby obtaining a microscopic derivation of the bulk entropy density. A salient aspect of the first-order rewriting that gives rise to these black branes is the fact that the angular momentum, the electric quantum numbers and the magnetic fields are organized into quantities which are invariant under the spectral flow of the theory, exactly as in the ungauged case [26]. This serves as a useful tool to identify the real quantum numbers of a worldvolume CFT in a string theory construction of gauged supergravity, and sets an indicator of the symmetries such a purported theory should satisfy.

We also reproduce the non-extremal black brane solutions of [27] and the electric solutions obtained recently in [7, 24].

The paper is organized as follows. We consider two first-order rewritings of the bosonic 
action of $N=2, \mathrm{U}(1)$ gauged supergravity. The first rewriting is performed in section 2 . The solution space of the resulting first-order flow equations encompasses static, purely magnetic solutions. We verify that the Hamiltonian constraint is satisfied (appendix A summarizes the Einstein equations of motion). In section 3 we briefly discuss the relation of these flow equations with the four-dimensional flow equations obtained in $[3,6]$. We refer to appendix $\mathrm{B}$ for the details of the comparison. In section 4 we turn to the construction of solutions to the first-order flow equations in five dimensions. First we consider exact solutions with constant scalar fields. These solutions do not carry electric fields, but may have magnetic fields, and they have rotation. We construct extremal $\mathrm{BTZ} \times \mathbb{R}^{2}$ solutions that are supported by magnetic fields, as well as rotating Nernst geometries in asymptotic $A d S_{5}$ backgrounds. Then we obtain numerical solutions with non-vanishing scalar fields, with and without rotation. These have $\mathrm{BTZ} \times \mathbb{R}^{2}$ near horizon geometry and are asymptotically $A d S_{5}$. They constitute generalizations of a solution given in [7] to the case with several running scalar fields and rotation.

In appendix $\mathrm{C}$ we turn to a different first-order rewriting. This is motivated by the search for solutions with electric fields. This rewriting is the one performed in [28] for static black hole solutions, which we adapt to the case of stationary black branes in the presence of magnetic fields. The resulting first-order flow equations allow for the nonextremal black brane solutions constructed in [27], as well as for the extremal electric solutions obtained in [7, 24].

\section{First-order flow equations for stationary solutions}

In the following, we derive first-order flow equations for extremal stationary black brane solutions in $N=2, \mathrm{U}(1)$ gauged supergravity in five dimensions with $n$ Abelian vector multiplets. We work in big moduli space. We follow the exposition given in [25] for the ungauged case and adapt it to the gauged case.

\subsection{Flow equations in big moduli space}

Following [21], we make the ansatz for the black brane line element,

$$
d s^{2}=-e^{2 U(r)} d t^{2}+e^{2 V(r)} d r^{2}+e^{2 B(r)}\left(d x^{2}+d y^{2}\right)+e^{2 W(r)}(d z+C(r) d t)^{2},
$$

while for the Abelian gauge fields $A_{M}^{A}(A=1, \ldots, n)$ we take

$$
A_{M}^{A} d x^{M}=A_{t}^{A} d t+P^{A} x d y+A_{z}^{A} d z=\left(e^{A}+A_{z}^{A} C(r)\right) d t+P^{A} x d y+A_{z}^{A} d z .
$$

Here the $P^{A}$ are constants and $A_{t}^{A}, A_{z}^{A}$ depend only on $r$. The associated field strength components read

$$
\begin{aligned}
& F_{r t}^{A}=\left(A_{t}^{A}\right)^{\prime}=\left(e^{A}\right)^{\prime}+A_{z}^{A} C^{\prime}+\left(A_{z}^{A}\right)^{\prime} C, \\
& F_{x y}^{A}=P^{A}, \\
& F_{r z}^{A}=\left(A_{z}^{A}\right)^{\prime},
\end{aligned}
$$


where ' denotes differentiation with respect to $r$, and $\left(e^{A}\right)^{\prime}$ corresponds to the fourdimensional electric field upon dimensional reduction. The solutions we seek will be supported by real scalar fields $X^{A}(r)$ and by electric fluxes $h_{A}$. The ansatz (2.1) and (2.2) is the most general ansatz with translational invariance in the coordinates $t, x, y$ and $z$ and with rotational invariance in the $x, y$-plane, cf. [15].

The bosonic part of the five-dimensional action describing $N=2, \mathrm{U}(1)$ gauged supergravity is given by $[29,30]$

$$
\begin{gathered}
S=\int d x^{5}\left[\sqrt { - g } \left(R-G_{A B} \partial_{M} X^{A} \partial^{M} X^{B}-\frac{1}{2} G_{A B} F_{M N}^{A} F^{B M N}\right.\right. \\
\left.-g^{2}\left(G^{A B} h_{A} h_{B}-2\left(h_{A} X^{A}\right)^{2}\right)\right) \\
\left.-\frac{1}{24} C_{A B C} F_{K L}^{A} F_{M N}^{B} A_{P}^{C} \epsilon^{K L M N P}\right]
\end{gathered}
$$

where the scalar fields $X^{A}$ satisfy the constraint $\frac{1}{6} C_{A B C} X^{A} X^{B} X^{C}=1$. The target space metric $G_{A B}$ is given by

$$
G_{A B}=-\frac{1}{2} C_{A B C} X^{C}+\frac{9}{2} X_{A} X_{B}
$$

where

$$
X_{A}=\frac{2}{3} G_{A B} X^{B}=\frac{1}{6} C_{A B C} X^{B} X^{C}
$$

Inserting the solution ansatz into this action, we find that the Ricci scalar contributes

$$
\begin{aligned}
\sqrt{-g} R= & e^{2 B+W+U-V}\left(2 B^{\prime 2}+2 U^{\prime} W^{\prime}+4 B^{\prime} W^{\prime}+4 B^{\prime} U^{\prime}+\frac{1}{2} e^{2 W-2 U} C^{\prime 2}\right) \\
& -\left[2 e^{2 B+W+U-V}\left(2 B^{\prime}+W^{\prime}+U^{\prime}\right)\right]^{\prime},
\end{aligned}
$$

while the gauge field kinetic terms contribute

$$
\begin{array}{r}
\sqrt{-g}\left(-\frac{1}{2} G_{A B} F_{M N}^{A} F^{B M N}\right)=e^{2 B+W+U-V}\left(-G_{A B} P^{A} P^{B} e^{2 V-4 B}+G_{A B} F_{r t}^{A} F_{r t}^{B} e^{-2 U}\right. \\
\left.-G_{A B}\left(A_{z}^{A}\right)^{\prime}\left(A_{z}^{B}\right)^{\prime}\left(e^{-2 W}-e^{-2 U} C^{2}\right)-2 G_{A B} F_{r t}^{A}\left(A_{z}^{B}\right)^{\prime} e^{-2 U} C\right),
\end{array}
$$

with $F_{r t}^{A}$ given in (2.3). The Chern-Simons term, on the other hand, can be rewritten as

$$
\int d x^{5}\left(-\frac{1}{24} C_{A B C} F_{K L}^{A} F_{M N}^{B} A_{P}^{C} \epsilon^{K L M N P}\right)=\int d x^{5}\left(-C_{A B C} F_{r t}^{A} F_{x y}^{B} A_{z}^{C}+T D\right)
$$

where $T D$ denotes a total derivative term. Inserting these expressions into (2.4) yields the 
one-dimensional Lagrangian $\mathcal{L}$,

$$
\begin{aligned}
& \mathcal{L}=e^{2 B+W+U-V}( 2 B^{\prime 2}+2 U^{\prime} W^{\prime}+4 B^{\prime} W^{\prime}+4 B^{\prime} U^{\prime}+\frac{1}{2} e^{2 W-2 U} C^{\prime 2}-G_{A B}\left(X^{A}\right)^{\prime}\left(X^{B}\right)^{\prime} \\
&-G_{A B} P^{A} P^{B} e^{2 V-4 B}+G_{A B}\left(e^{A}\right)^{\prime}\left(e^{B}\right)^{\prime} e^{-2 U}+G_{A B} A_{z}^{A} A_{z}^{B} C^{\prime 2} e^{-2 U} \\
&+2 G_{A B}\left(e^{A}\right)^{\prime} A_{z}^{B} C^{\prime} e^{-2 U}-G_{A B}\left(A_{z}^{A}\right)^{\prime}\left(A_{z}^{B}\right)^{\prime} e^{-2 W} \\
&\left.-g^{2} e^{2 V}\left(G^{A B} h_{A} h_{B}-2\left(h_{A} X^{A}\right)^{2}\right)\right) \\
&-C_{A B C}\left(e^{A}\right)^{\prime} P^{B} A_{z}^{C}-C_{A B C} A_{z}^{A} P^{B} A_{z}^{C} C^{\prime}-C_{A B C}\left(A_{z}^{A}\right)^{\prime} P^{B} A_{z}^{C} C
\end{aligned}
$$

where we dropped total derivative terms.

Now we express the electric field $\left(e^{A}\right)^{\prime}$ in terms of electric charges $q_{A}$ by performing the Legendre transformation $\mathcal{L}_{L}=\mathcal{L}-q_{A}\left(e^{A}\right)^{\prime}$, and obtain

$$
\left(e^{A}\right)^{\prime}=\frac{1}{2} e^{-2 B-W+U+V} G^{A B} \hat{q}_{B}-A_{z}^{A} C^{\prime},
$$

where

$$
\hat{q}_{A}=q_{A}+C_{A B C} P^{B} A_{z}^{C} .
$$

Substituting this relation in (2.10) gives

$$
\begin{aligned}
& \mathcal{L}=e^{2 B+W+U-V}( 2 B^{2}+2 U^{\prime} W^{\prime}+4 B^{\prime} W^{\prime}+4 B^{\prime} U^{\prime}+\frac{1}{2} e^{2 W-2 U} C^{2}-G_{A B}\left(X^{A}\right)^{\prime}\left(X^{B}\right)^{\prime} \\
&-G_{A B} P^{A} P^{B} e^{2 V-4 B}-\frac{1}{4} G^{A B} \hat{q}_{A} \hat{q}_{B} e^{-4 B-2 W+2 V} \\
&\left.-G_{A B}\left(A_{z}^{A}\right)^{\prime}\left(A_{z}^{B}\right)^{\prime} e^{-2 W}-g^{2} e^{2 V}\left(G^{A B} h_{A} h_{B}-2\left(h_{A} X^{A}\right)^{2}\right)\right) \\
&-C_{A B C}\left(A_{z}^{A}\right)^{\prime} P^{B} A_{z}^{C} C+q_{A} A_{z}^{A} C^{\prime}
\end{aligned}
$$

Furthermore, using

$$
\left(C_{A B C} A_{z}^{A} P^{B} A_{z}^{C} C\right)^{\prime}=2 C_{A B C}\left(A_{z}^{A}\right)^{\prime} P^{B} A_{z}^{C} C+C_{A B C} A_{z}^{A} P^{B} A_{z}^{C} C^{\prime},
$$

we obtain

$$
-C_{A B C}\left(A_{z}^{A}\right)^{\prime} P^{B} A_{z}^{C} C=\frac{1}{2} C_{A B C} A_{z}^{A} P^{B} A_{z}^{C} C^{\prime}+T D,
$$

where $T D$ denotes again a total derivative, which we drop in the following.

Next, we express $C^{\prime}$ in terms of a constant quantity $J$ which, in the compact case, corresponds to angular momentum. We do this by performing the Legendre transformation $\mathcal{L}_{L}=\mathcal{L}-J C^{\prime}$, and obtain

$$
C^{\prime}=e^{-2 B-3 W+U+V} \hat{J}
$$

where

$$
\hat{J}=J-q_{A} A_{z}^{A}-\frac{1}{2} C_{A B C} A_{z}^{A} A_{z}^{B} P^{C} .
$$


This results in

$$
\begin{aligned}
\mathcal{L}= & e^{2 B+W+U-V}\left(2 B^{\prime 2}+2 U^{\prime} W^{\prime}+4 B^{\prime} W^{\prime}+4 B^{\prime} U^{\prime}-G_{A B}\left(X^{A}\right)^{\prime}\left(X^{B}\right)^{\prime}\right. \\
& -G_{A B}\left(A_{z}^{A}\right)^{\prime}\left(A_{z}^{B}\right)^{\prime} e^{-2 W}-G_{A B} P^{A} P^{B} e^{2 V-4 B}-\frac{1}{4} G^{A B} \hat{q}_{A} \hat{q}_{B} e^{-4 B-2 W+2 V} \\
& \left.-\frac{1}{2} e^{-4 B-4 W+2 V} \hat{J}^{2}-g^{2} e^{2 V}\left(G^{A B} h_{A} h_{B}-2\left(h_{A} X^{A}\right)^{2}\right)\right) .
\end{aligned}
$$

Now we rewrite the one-dimensional Lagrangian (2.18) as a sum of squares of first-order flow equations. To this end, we use the relation

$$
\left(e^{U-W}\left(\hat{q}_{A} A_{z}^{A}-\frac{1}{2} C_{A B C} A_{z}^{A} P^{B} A_{z}^{C}\right)\right)^{\prime}=e^{U-W}\left(\left(U^{\prime}-W^{\prime}\right)(-\hat{J})+\hat{q}_{A}\left(A_{z}^{A}\right)^{\prime}\right)+\left(J e^{U-W}\right)^{\prime},
$$

and obtain

$$
\begin{aligned}
\mathcal{L}=e^{2 B+W+U-V}[ & -e^{-2 W} G_{A B}\left(\left(A_{z}^{A}\right)^{\prime}+\frac{1}{2} G^{A C} \hat{q}_{C} e^{-2 B+V}\right)\left(\left(A_{z}^{B}\right)^{\prime}+\frac{1}{2} G^{B D} \hat{q}_{D} e^{-2 B+V}\right) \\
& -\frac{1}{2}\left(\hat{J} e^{-2 B-2 W+V}-\left(U^{\prime}-W^{\prime}\right)\right)^{2} \\
& -\left(B^{\prime}-\frac{1}{2}\left(U^{\prime}+W^{\prime}\right)+\frac{3}{2} X_{A} P^{A} e^{V-2 B}\right)^{2} \\
& +\frac{1}{3}\left(3\left(B^{\prime}+\frac{1}{2}\left(U^{\prime}+W^{\prime}\right)\right)-2 g X^{A} h_{A} e^{V}+\frac{3}{2} X_{A} P^{A} e^{V-2 B}\right)^{2} \\
& -G_{A B}\left(X^{\prime A}-e^{V}\left[\frac{2}{3} X^{C}\left(g h_{C}+G_{C D} P^{D} e^{-2 B}\right) X^{A}-G^{A C}\left(g h_{C}+G_{C D} P^{D} e^{-2 B}\right)\right]\right) \\
& \left.\left(X^{\prime B}-e^{V}\left[\frac{2}{3} X^{E}\left(g h_{E}+G_{E F} P^{F} e^{-2 B}\right) X^{B}-G^{B E}\left(g h_{E}+G_{E F} P^{F} e^{-2 B}\right)\right]\right)\right] \\
+ & 2\left(e^{2 B+W+U}\left(g X^{A} h_{A}-\frac{3}{2} X_{A} P^{A} e^{-2 B}\right)\right)^{\prime} \\
+ & \left(e^{U-W}\left(\hat{q}_{A} A_{z}^{A}-\frac{1}{2} C_{A B C} A_{z}^{A} P^{B} A_{z}^{C}\right)\right)^{\prime}-\left(J e^{U-W}\right)^{\prime} \\
+ & 2 g e^{W+U+V} h_{A} P^{A} .
\end{aligned}
$$

This concludes the rewriting of the effective one-dimensional Lagrangian.

Setting the squares in (2.20) to zero yields the first-order flow equations

$$
\begin{aligned}
\left(A_{z}^{A}\right)^{\prime} & =-\frac{1}{2} G^{A C} \hat{q}_{C} e^{V-2 B}, \\
\left(X^{A}\right)^{\prime} & =\frac{2}{3} X^{C}\left(g h_{C} e^{V}+G_{C D} P^{D} e^{V-2 B}\right) X^{A}-G^{A C}\left(g h_{C} e^{V}+G_{C D} P^{D} e^{V-2 B}\right), \\
U^{\prime}-W^{\prime} & =\hat{J} e^{-2 B-2 W+V} \\
0 & =B^{\prime}-\frac{1}{2}\left(U^{\prime}+W^{\prime}\right)+\frac{3}{2} X_{A} P^{A} e^{V-2 B}, \\
0 & =3\left(B^{\prime}+\frac{1}{2}\left(U^{\prime}+W^{\prime}\right)\right)-2 g X^{A} h_{A} e^{V}+\frac{3}{2} X_{A} P^{A} e^{V-2 B} .
\end{aligned}
$$


These flow equations are supplemented by (2.11) and (2.16), and solutions to these equations are subjected to the constraint

$$
h_{A} P^{A}=0
$$

which follows from the last line of (2.20). Note that the flow equations (2.21) show an interesting decoupling: the scalar fields $X^{A}$ and the metric coefficient $e^{B}$ are completely determined by the magnetic fields and the fluxes, whereas the electric charges only enter in the equations for the metric functions $e^{U}$ and $e^{W}$ and the $A_{z}^{A}$-components of the gauge fields. This will be helpful in the search for solutions, cf. section 4 .

Subtracting the fourth from the fifth equation in (2.21) gives

$$
B^{\prime}+U^{\prime}+W^{\prime}=g h_{A} X^{A} e^{V} .
$$

When $B$ is constant, this yields a flow equation for $U+W$ that, when compared with the fourth equation of (2.21), yields the condition

$$
g X^{A} h_{A}=3 X_{A} P^{A} e^{-2 B} .
$$

Also observe that (2.3), (2.11) and the first equation of (2.21) implies

$$
F_{r t}^{A}=\frac{1}{2}(1-\hat{C}) e^{-2 B-W+U+V} G^{A B} \hat{q}_{B},
$$

where

$$
\hat{C} \equiv C e^{-(U-W)}
$$

while the third equation, together with (2.16), gives

$$
C^{\prime}=\left(U^{\prime}-W^{\prime}\right) e^{U-W}=\left(e^{U-W}\right)^{\prime},
$$

and hence

$$
\hat{C}=1+\lambda e^{-(U-W)},
$$

with $\lambda$ a real integration constant. ${ }^{1}$ Inserting this into (2.25) gives

$$
F_{r t}^{A}=-\frac{\lambda}{2} e^{-2 B+V} G^{A B} \hat{q}_{B} .
$$

However, the electric field is actually given by

$$
\left(F^{A}\right)^{t r}=F_{t r}^{A} g^{t t} g^{r r}+F_{z r}^{A} g^{t z} g^{r r}=\frac{1}{2} e^{-2 B-W-U-V} G^{A B} \hat{q}_{B}
$$

where we used the form of the inverse metric, the third equation of (2.3), together with the first equation of (2.21), and (2.29). Comparing this with (2.11), we see that this can also be expressed as

$$
\left(F^{A}\right)^{t r}=e^{-2 U-2 V}\left(\left(e^{A}\right)^{\prime}+A_{z}^{A} C^{\prime}\right) .
$$

\footnotetext{
${ }^{1}$ Given the relation $C=e^{U-W}+\lambda$ between three of the metric functions, the solution set of the first-order equations (2.21) is naturally more restricted than the one obtained by looking at the second order equations of motion. In particular, the charged magnetic brane solution of $[12,15]$ is not a solution of $(2.21)$.
} 
Obviously, the electric field is independent of the integration constant $\lambda$ and is nonvanishing whenever some of the charges $\hat{q}_{A}$ are non-vanishing.

In contrast, the five-dimensional magnetic field component $\left(F^{A}\right)^{r z}$ does depend on $\lambda$ according to

$$
\left(F^{A}\right)^{r z}=F_{r z}^{A} g^{r r} g^{z z}+F_{r t}^{A} g^{r r} g^{t z}=\frac{1}{2} \lambda e^{-2 B-W-U-V} G^{A B} \hat{q}_{B} .
$$

Notice, however, that both the electric field and the $r z$-component of the magnetic field are determined by the charges $\hat{q}_{A}$. As a consequence, the combination $G_{A B} F_{M N}^{A} F^{B M N}$ vanishes (independently of $\lambda$ ) for vanishing $P^{A}$ on any solution of (2.21), i.e.

$$
G_{A B} F_{r t}^{A} F^{B r t}=-G_{A B} F_{r z}^{A} F^{B r z} .
$$

On the other hand, inserting (2.28) into the line element (2.1) results in

$$
d s^{2}=e^{2 W} \lambda\left(\lambda+2 e^{U-W}\right) d t^{2}+2 e^{2 W} C d t d z+e^{2 W} d z^{2}+e^{2 V} d r^{2}+e^{2 B}\left(d x^{2}+d y^{2}\right) .
$$

Thus, we see that the sign and the magnitude of the integration constant $\lambda$ determine the nature of the warped line element. In particular, a vanishing $\lambda$ will give a null-warped metric, i.e. $g_{t t}=0$.

Let us now briefly display the flow equations for static, purely magnetic solutions. They are obtained by setting $q_{A}=A_{z}^{A}=J=0$, which results in $\hat{q}_{A}=\hat{J}=C^{\prime}=0$, so that the non-vanishing flow equations are

$$
\begin{aligned}
\left(X^{A}\right)^{\prime} & =\frac{2}{3} X^{C}\left(g h_{C} e^{V}+G_{C D} P^{D} e^{V-2 B}\right) X^{A}-G^{A C}\left(g h_{C} e^{V}+G_{C D} P^{D} e^{V-2 B}\right), \\
U^{\prime}-W^{\prime} & =0 \\
0 & =B^{\prime}-\frac{1}{2}\left(U^{\prime}+W^{\prime}\right)+\frac{3}{2} X_{A} P^{A} e^{V-2 B}, \\
0 & =3\left(B^{\prime}+\frac{1}{2}\left(U^{\prime}+W^{\prime}\right)\right)-2 g X^{A} h_{A} e^{V}+\frac{3}{2} X_{A} P^{A} e^{V-2 B} .
\end{aligned}
$$

These flow equations need again to be supplemented by the constraint $h_{A} P^{A}=0$. Magnetic supersymmetric $A d S_{3} \times \mathbb{R}^{2}$ solutions to these equations were studied in [7, 19, 22, 23].

Finally, we would like to show that the flow equations (2.21) follow from a superpotential. To do so, it is convenient to introduce the combinations

$$
\phi_{1}=B-\frac{1}{2}(U+W), \quad \phi_{2}=B+\frac{1}{2}(U+W), \quad \phi_{3}=U-W .
$$

Using them and introducing the physical scalars $\varphi^{i}$, the one-dimensional Lagrangian (2.18) takes the form

$$
\begin{aligned}
\mathcal{L}= & -e^{2 \phi_{2}-V}\left(\phi_{1}^{\prime}\right)^{2}+3 e^{2 \phi_{2}-V}\left(\phi_{2}^{\prime}\right)^{2}-\frac{1}{2} e^{2 \phi_{2}-V}\left(\phi_{3}^{\prime}\right)^{2} \\
& -e^{2 \phi_{2}-V} G_{i j}\left(\varphi^{i}\right)^{\prime}\left(\varphi^{j}\right)^{\prime}-e^{\phi_{1}+\phi_{2}+\phi_{3}-V} G_{A B}\left(A_{z}^{A}\right)^{\prime}\left(A_{z}^{B}\right)^{\prime} \\
& -e^{-2 \phi_{1}+V} G_{A B} P^{A} P^{B}-\frac{1}{4} e^{-\phi_{1}-\phi_{2}+\phi_{3}+V} G^{A B} \hat{q}_{A} \hat{q}_{B} \\
& -\frac{1}{2} e^{-2 \phi_{2}+2 \phi_{3}+V} \hat{J}^{2}-g^{2} e^{2 \phi_{2}+V}\left(G^{A B} h_{A} h_{B}-2\left(h_{A} X^{A}\right)^{2}\right),
\end{aligned}
$$


where we used $[29,30]$

$$
G_{i j}=G_{A B} \partial_{i} X^{A} \partial_{j} X^{B}
$$

The advantage of working with the combinations (2.36) is that the sigma-model metric is then block diagonal with

$$
\begin{aligned}
g_{\phi_{1} \phi_{1}} & =e^{2 \phi_{2}-V}, & g_{\phi_{2} \phi_{2}} & =-3 e^{2 \phi_{2}-V},
\end{aligned} g_{\phi_{3} \phi_{3}}=\frac{1}{2} e^{2 \phi_{2}-V},
$$

It is now a straightforward exercise to show that the potential $\mathcal{V}$ of the one-dimensional Lagrangian (i.e. the last two lines of (2.37)) can be expressed as

$$
\mathcal{V}=-g^{\phi_{1} \phi_{1}}\left(\frac{\partial \mathcal{Z}}{\partial \phi_{1}}\right)^{2}-g^{\phi_{2} \phi_{2}}\left(\frac{\partial \mathcal{Z}}{\partial \phi_{2}}\right)^{2}-g^{\phi_{3} \phi_{3}}\left(\frac{\partial \mathcal{Z}}{\partial \phi_{3}}\right)^{2}-g^{i j} \frac{\partial \mathcal{Z}}{\partial \varphi^{i}} \frac{\partial \mathcal{Z}}{\partial \varphi^{j}}-g^{A B} \frac{\partial \mathcal{Z}}{\partial A_{z}^{A}} \frac{\partial \mathcal{Z}}{\partial A_{z}^{B}}
$$

with the superpotential

$$
\mathcal{Z}=\frac{1}{2} e^{\phi_{3}} \hat{J}+\frac{3}{2} e^{\phi_{2}-\phi_{1}} P^{A} X_{A}-e^{2 \phi_{2}} g h_{A} X^{A}
$$

In doing so, one has to make use of the constraint $h_{A} P^{A}=0$, of $(2.38)$ and $[29,30]$

$$
G^{i j} \partial_{i} X^{A} \partial_{j} X^{B}=G^{A B}-\frac{2}{3} X^{A} X^{B}, \quad \partial_{i} X_{A}=-\frac{2}{3} G_{A B} \partial_{i} X^{B}, \quad X_{A}=\frac{2}{3} G_{A B} X^{B} .
$$

Using the superpotential (2.41), it is straightforward to check that the first-order flow equations (in the physical moduli space) can be expressed $\mathrm{as}^{2}$

$$
\begin{aligned}
& \phi_{1}^{\prime}=g^{\phi_{1} \phi_{1}} \frac{\partial \mathcal{Z}}{\partial \phi_{1}}, \quad \quad \phi_{2}^{\prime}=g^{\phi_{2} \phi_{2}} \frac{\partial \mathcal{Z}}{\partial \phi_{2}}, \quad \phi_{3}^{\prime}=g^{\phi_{3} \phi_{3}} \frac{\partial \mathcal{Z}}{\partial \phi_{3}}, \\
& \left(A_{z}^{A}\right)^{\prime}=g^{A B} \frac{\partial \mathcal{Z}}{\partial A_{z}^{B}}, \quad\left(\varphi^{i}\right)^{\prime}=g^{i j} \frac{\partial \mathcal{Z}}{\partial \varphi^{j}} .
\end{aligned}
$$

In order to derive the flow equation for $\varphi^{i}$, one has to multiply the flow equation for $X^{A}$ by $G_{A B} \partial_{j} X^{B}$ and use (2.38), (2.42) and

$$
X_{A} \partial_{i} X^{A}=0 .
$$

This leads to

$$
\left(\varphi^{i}\right)^{\prime}=-G^{i j} e^{V}\left(g h_{A}+G_{A C} P^{C} e^{-2 B}\right) \partial_{j} X^{A}
$$

In the absence of fluxes, the superpotential (2.41) reduces to the one obtained in [25].

\footnotetext{
${ }^{2}$ Note that (2.40) would hold also for any combination of signs in $\mathcal{Z}=\frac{1}{2} e^{\phi_{3}} \hat{J} \pm \frac{3}{2} e^{\phi_{2}-\phi_{1}} P^{A} X_{A} \pm$ $e^{2 \phi_{2}} g h_{A} X^{A}$, but (2.43) requires the signs given in (2.41).
} 


\subsection{Hamiltonian constraint}

Next, we discuss the Hamiltonian constraint and show that it equals the constraint $h_{A} P^{A}=0$ that we encountered in the rewriting of the Lagrangian in terms of first-order flow equations.

The Einstein equations take the form

$$
\begin{aligned}
R_{M N}= & \frac{1}{3} g_{M N} g^{2}\left(G^{A B} h_{A} h_{B}-2\left(h_{A} X^{A}\right)^{2}\right)-\frac{1}{6} g_{M N} G_{A B} F_{K L}^{A} F^{B K L} \\
& +G_{A B}\left(X^{A}\right)^{\prime}\left(X^{B}\right)^{\prime} \delta_{M r} \delta_{N r}+G_{A B} F_{M K}^{A} F_{N L}^{B} g^{K L} .
\end{aligned}
$$

There are only five independent equations, namely the ones corresponding to the $t t_{-}, \mathrm{rr}-$, $x x-, z z$ - and $t z$-component of the Ricci tensor, which we have displayed in appendix A. To obtain the Hamiltonian constraint, we consider the $t t$-component of Einstein's equations. We use the $r r_{-}, x x-, z z$ - and $t z$-equations to obtain expressions for the second derivatives $U^{\prime \prime}, C^{\prime \prime}, B^{\prime \prime}$ and $W^{\prime \prime}$, which we then insert into the expression for the $t t$-component. This yields the following equation, which now only contains first derivatives,

$$
\begin{aligned}
0= & 2 B^{\prime 2}+2 U^{\prime} W^{\prime}+4 B^{\prime} W^{\prime}+4 B^{\prime} U^{\prime}+\frac{1}{2} e^{2 W-2 U} C^{2}-G_{A B}\left(X^{A}\right)^{\prime}\left(X^{B}\right)^{\prime}+G_{A B} F_{r t}^{A} F_{r t}^{B} e^{-2 U} \\
& +G_{A B} P^{A} P^{B} e^{2 V-4 B}-G_{A B}\left(A_{z}^{A}\right)^{\prime}\left(A_{z}^{B}\right)^{\prime}\left(e^{-2 W}-e^{-2 U} C^{2}\right)-2 G_{A B} F_{r t}^{A}\left(A_{z}^{B}\right)^{\prime} e^{-2 U} C \\
& +g^{2} e^{2 V}\left(G^{A B} h_{A} h_{B}-2\left(h_{A} X^{A}\right)^{2}\right) .
\end{aligned}
$$

This equation turns out to be equivalent to the $r r$-component of Einstein's equations,

$$
R_{r r}-\frac{1}{2} g_{r r} R-\frac{1}{2} g_{r r} \mathcal{L}_{M}+\frac{\delta \mathcal{L}_{M}}{\delta g^{r r}}=0,
$$

where $\mathcal{L}_{M}$ denotes the matter Lagrangian.

Next, using $(2.11),(2.16),(2.27)$ and the flow equation for $\left(A_{z}^{A}\right)^{\prime}$ in $(2.47)$, we obtain the intermediate result

$$
\begin{aligned}
0= & 2 B^{\prime 2}+2 U^{\prime} W^{\prime}+4 B^{\prime} W^{\prime}+4 B^{\prime} U^{\prime}+\frac{1}{2}\left(U^{\prime}-W^{\prime}\right)^{2}-G_{A B}\left(X^{A}\right)^{\prime}\left(X^{B}\right)^{\prime} \\
& +G_{A B} P^{A} P^{B} e^{2 V-4 B}+g^{2} e^{2 V}\left(G^{A B} h_{A} h_{B}-2\left(h_{A} X^{A}\right)^{2}\right) .
\end{aligned}
$$

Then, using the first-order flow equation $(2.21)$ for $\left(X^{A}\right)^{\prime}$, we get

$$
\begin{aligned}
0= & 2 B^{\prime 2}+2 U^{\prime} W^{\prime}+4 B^{\prime} W^{\prime}+4 B^{\prime} U^{\prime}+\frac{1}{2}\left(U^{\prime}-W^{\prime}\right)^{2}-\frac{4}{3} g^{2} e^{2 V}\left(h_{A} X^{A}\right)^{2} \\
& +\frac{2}{3}\left(G_{A B} X^{A} P^{B}\right)^{2} e^{2 V-4 B}+\frac{4}{3} g e^{2 V-2 B}\left(h_{A} X^{A}\right)\left(G_{A B} X^{A} P^{B}\right)-2 g e^{2 V-2 B} h_{A} P^{A} .
\end{aligned}
$$

In the next step we use (2.23) as well as the fourth flow equation of (2.21) to obtain

$$
\begin{aligned}
0= & 2 B^{\prime 2}+2 U^{\prime} W^{\prime}+4 B^{\prime} W^{\prime}+4 B^{\prime} U^{\prime}+\frac{1}{2}\left(U^{\prime}-W^{\prime}\right)^{2}-\frac{4}{3}\left(B^{\prime}+U^{\prime}+W^{\prime}\right)^{2} \\
& +\frac{2}{3}\left(-B^{\prime}+\frac{1}{2}\left(U^{\prime}+W^{\prime}\right)\right)^{2}+\frac{4}{3}\left(B^{\prime}+U^{\prime}+W^{\prime}\right)\left(-B^{\prime}+\frac{1}{2}\left(U^{\prime}+W^{\prime}\right)\right) \\
& -2 g e^{2 V-2 B} h_{A} P^{A} .
\end{aligned}
$$

Then, one checks that all the terms containing $B^{\prime}, U^{\prime}$ and $W^{\prime}$ cancel out, so that the on-shell Hamiltonian constraint (2.51) reduces to (2.22). 


\section{$3 \quad$ Reducing to four dimensions}

The five-dimensional stationary solutions to the flow equations (2.21) may be related to a subset of the four-dimensional static solutions discussed in $[3,6]$ by performing a reduction on the $z$-direction. We briefly describe this below. A detailed check of the matching of the five- and four-dimensional flow equations is performed in appendix B.

The five-dimensional solutions are supported by electric fluxes $h_{A}^{5 \mathrm{~d}}$, electric charges $q_{A}^{5 \mathrm{~d}}$, magnetic fields $P_{5 \mathrm{~d}}^{A}$, and rotation $J .^{3}$ The relevant subset of four-dimensional solutions is supported by electric fluxes $h_{A}^{4 \mathrm{~d}}$, electric charges $Q_{I}=\left(Q_{0}, Q_{A}\right)$ and magnetic fields $P_{4 \mathrm{~d}}^{A}$.

The five-dimensional $N=2, \mathrm{U}(1)$ gauged supergravity action (2.4) is based on real scalar fields $X_{5 \mathrm{~d}}$ which satisfy the constraint $\frac{1}{6} C_{A B C} X_{5 \mathrm{~d}}^{A} X_{5 \mathrm{~d}}^{B} X_{5 \mathrm{D}}^{C}=1$ for some constants $C_{A B C}$, while the four-dimensional $N=2, \mathrm{U}(1)$ gauged supergravity action considered in $[3,6]$ is based on complex scalar fields $X_{4 \mathrm{D}}^{I}$ with a cubic prepotential function

$$
F\left(X_{4 \mathrm{~d}}\right)=-\frac{1}{6} \frac{C_{A B C} X_{4 \mathrm{~d}}^{A} X_{4 \mathrm{~d}}^{B} X_{4 \mathrm{~d}}^{C}}{X_{4 \mathrm{~d}}^{0}}
$$

The four-dimensional physical scalar fields are $z^{A}=X_{4 \mathrm{~d}}^{A} / X_{4 \mathrm{~d}}^{0}$, which we decompose as $z^{A}=C^{A}+i \hat{X}^{A}$.

Now we relate the real four-dimensional fields $\left(C^{A}, \hat{X}^{A}\right)$ to the fields appearing in the five-dimensional flow equations. To do so, we find it convenient to use a different normalization for the scalar constraint equation, namely

$$
\frac{1}{6} C_{A B C} X_{5 \mathrm{~d}}^{A} X_{5 \mathrm{~d}}^{B} X_{5 \mathrm{D}}^{C}=v
$$

We will show in the appendix that the matching between the four-dimensional and the fivedimensional flow equations requires to choose $v=\frac{1}{2}$, a value which was already obtained in [31] when matching the gauge kinetic terms in four and five dimensions. Choosing the normalization (3.2) amounts to replacing $C_{A B C}$ by $C_{A B C} / v$, a change that affects the normalization of the Chern-Simons term in the five-dimensional action (2.4), as well as the quantities $\hat{q}_{A}$ and $\hat{J}$ given in (2.12) and (2.17), respectively. On the other hand, if we stick to the definition $X_{A}^{5 \mathrm{~d}}=\frac{1}{6} C_{A B C} X_{5 \mathrm{~d}}^{B} X_{5 \mathrm{~d}}^{C}$, we get

$$
X_{A}^{5 \mathrm{~d}}=\frac{2 v}{3} G_{A B} X_{5 \mathrm{~d}}^{B}
$$

with $G_{A B}$ given by

$$
G_{A B}\left(X_{5 \mathrm{~d}}\right)=\frac{1}{v}\left(-\frac{1}{2} C_{A B C} X_{5 \mathrm{~d}}^{C}+\frac{9}{2 v} X_{A}^{5 \mathrm{~d}} X_{B}^{5 \mathrm{~d}}\right)
$$

\footnotetext{
${ }^{3} J$ generates translations in the $z$-direction. When the coordinate $z$ is compact, $J$ has the interpretation of angular momentum.
} 
Using this normalization, we obtain the following dictionary between the four-dimensional quantities that appeared in [6] and the five-dimensional quantities that enter in (2.21),

$$
\begin{aligned}
\hat{X}^{A} & =e^{W} X_{5 \mathrm{~d}}^{A}, \\
C^{A} & =A_{z}^{A}, \\
h_{A}^{4 \mathrm{~d}} & =-h_{A}^{5 \mathrm{~d}}, \\
P_{4 \mathrm{~d}}^{A} & =-P_{5 \mathrm{~d}}^{A}, \\
Q_{A}^{4 \mathrm{~d}} & =-\frac{1}{2} q_{A}^{5 \mathrm{~d}}, \\
Q_{0}^{4 \mathrm{~d}} & =\frac{1}{2} J .
\end{aligned}
$$

The five- and four-dimensional line elements are related by

$$
d s_{5}^{2}=e^{2 \phi} d s_{4}^{2}+e^{-4 \phi}(d z+C d t)^{2}
$$

where

$$
d s_{4}^{2}=-e^{2 U_{4}} d t^{2}+e^{-2 U_{4}} d r^{2}+e^{-2 U_{4}+2 \psi}\left(d x^{2}+d y^{2}\right)
$$

and

$$
2 \phi=-W
$$

This yields

$$
\begin{aligned}
& U=U_{4}+\phi, \\
& V=-U_{4}+\phi, \\
& B=\psi-U_{4}+\phi .
\end{aligned}
$$

\section{Solutions}

In the following, we construct solutions to the flow equations (2.21). First we consider exact solutions with constant scalars $X^{A}$. Subsequently we numerically construct solutions with running scalars $X^{A}$.

\subsection{Solutions with constant scalar fields $X^{A}$}

We pick $V=0$ in the following.

We will consider two distinct cases. In the first case, all the magnetic fields $P^{A}$ are taken to be non-vanishing. In the second case, we set all the $P^{A}$ to zero. Other cases where only some of the $P^{A}$ are turned on are also possible, and their analysis should go along similar lines. 


\subsubsection{Taking $P^{A} \neq 0, \hat{q}_{A}=0, \hat{J} \neq 0$}

Here we consider the case when all the $P^{A}$ are turned on. Demanding $X^{A}=$ constant yields

$$
X^{C}\left(g h_{C}+G_{C D} P^{D} e^{-2 B}\right) X_{A}=g h_{A}+G_{A B} P^{B} e^{-2 B} .
$$

Observe that $G_{A B}$ is constant, and so is $B$. We set $B=0$ in the following, which can always be achieved by rescaling $x$ and $y$. Combining (4.1) with (2.24), we express the magnetic fields $P^{A}$ in terms of $h_{A}$ and $X^{A}$ as

$$
P^{A}=-g G^{A B}\left(h_{B}-\frac{3}{2}\left(h_{C} X^{C}\right) X_{B}\right) .
$$

This relation generically fixes the scalars $X^{A}=X^{A}\left(h_{B}, P^{B}\right)$ in terms of the fluxes and magnetic fields, as we will see in the explicit examples of section 4.2. Contracting (4.2) with $h_{A}$ and using the constraint (2.22) we obtain

$$
G^{A B} h_{A} h_{B}=\left(h_{A} X^{A}\right)^{2}
$$

as well as

$$
G_{A B} P^{A} P^{B}=\frac{1}{2} g^{2}\left(h_{A} X^{A}\right)^{2} .
$$

Observe that (4.3) together with $h_{A} X^{A}=0$ would imply $h_{A}=0$. Thus, in the following, we take $h_{A} X^{A} \neq 0$.

We obtain from (2.23),

$$
U+W=g h_{A} X^{A}\left(r-r_{0}\right),
$$

where $r_{0}$ denotes an integration constant. Inserting this into the third equation of $(2.21)$ gives

$$
\left(e^{-(U-W)}\right)^{\prime}=-\hat{J} e^{g h_{A} X^{A}\left(r_{0}-r\right)} .
$$

Next we set $\hat{q}_{A}=0$, so that the $A_{z}^{A}$ take constant values. These are determined by

$$
q_{A}+C_{A B C} P^{B} A_{z}^{C}=0 .
$$

Defining $C_{A B}=C_{A B C} P^{C}$, this is solved by

$$
A_{z}^{A}=-C^{A B} q_{B}
$$

where $C^{A B} C_{B C}=\delta^{A}{ }_{C}$. Here, we assumed that $C_{A B}$ is invertible, which generically is the case when all the $P^{A}$ are turned on.

For constant $A_{z}^{A}, \hat{J}$ is also constant, and we can solve (4.6). Taking $h_{A} X^{A} \neq 0$, we get

$$
e^{-(U-W)}=\frac{\hat{J} e^{g h_{A} X^{A}\left(r_{0}-r\right)}}{g h_{A} X^{A}}+b
$$

where $b$ denotes an integration constant. Combining this result with (4.5) gives

$$
e^{2 W}=\frac{\hat{J}}{g h_{A} X^{A}}+b e^{g h_{A} X^{A}\left(r-r_{0}\right)}
$$


as well as

$$
e^{-2 U}=\frac{\hat{J} e^{2 g h_{A} X^{A}\left(r_{0}-r\right)}}{g h_{A} X^{A}}+b e^{g h_{A} X^{A}\left(r_{0}-r\right)} .
$$

To bring these expressions into a more palatable form, we introduce a new radial variable

$$
\tau=\alpha e^{g h_{A} X^{A}\left(r-r_{0}\right)}
$$

with $\tau \geq 0$ and

$$
\alpha=g h_{A} X^{A}>0 .
$$

(If $g h_{A} X^{A}<0$ we have $\tau \leq 0$.) Then (assuming $\hat{J} \geq 0$ )

$$
\begin{aligned}
e^{2 W} & =\alpha^{-1}[\hat{J}+b \tau], \\
e^{-2 U} & =\alpha\left[\frac{\hat{J}}{\tau^{2}}+\frac{b}{\tau}\right], \\
C & =\frac{\tau}{\hat{J}+b \tau}+\lambda,
\end{aligned}
$$

and the associated line element reads

$$
\begin{aligned}
d s^{2}= & -\alpha^{-1} \frac{\tau^{2}}{\hat{J}+b \tau} d t^{2}+\frac{\alpha^{-2}}{\tau^{2}} d \tau^{2} \\
& +\alpha^{-1}(\hat{J}+b \tau)\left(d z+\left[\frac{\tau}{\hat{J}+b \tau}+\lambda\right] d t\right)^{2} \\
& +\left(d x^{2}+d y^{2}\right)
\end{aligned}
$$

Now we notice that for

$$
\lambda=-\frac{1}{b} \text { and } b=4 \alpha^{-3}>0
$$

and assuming $z$ to be compact, this is nothing but the metric of the extremal BTZ black hole in $A d S_{3}$ times $\mathbb{R}^{2}$, so that the space time is asymptotically $A d S_{3} \times \mathbb{R}^{2}$. This can be made manifest by the coordinate redefinitions

$$
\tau=\rho^{2}-\frac{\hat{J}}{b}, \quad z=\frac{l}{b} \phi,
$$

where $l^{2}=\alpha b=4 /\left(g h_{A} X^{A}\right)^{2}$. Introducing

$$
j=\frac{2 \hat{J}}{b l}
$$

the line element becomes

$$
d s^{2}=-\left(\frac{\rho}{l}-\frac{j}{2 \rho}\right)^{2} d t^{2}+\left(\frac{\rho}{l}-\frac{j}{2 \rho}\right)^{-2} d \rho^{2}+\rho^{2}\left(d \phi-\frac{j}{2 \rho^{2}} d t\right)^{2}+\left(d x^{2}+d y^{2}\right) .
$$

This describes an extremal BTZ black hole with angular momentum $j$ and mass $M=$ $j / l[32]$, where $l$ denotes the radius of $A d S_{3}$. The horizon is at $\rho_{+}^{2}=j l / 2=\hat{J} / b$, which 
corresponds to $\tau=0$. The entropy of the BTZ black hole (and hence the entropy density of the extremal BTZ $\times \mathbb{R}^{2}$ solution (4.19)) is

$$
\mathcal{S}_{\mathrm{BTZ}}=\frac{2 \pi \rho_{+}}{4}=\pi \frac{\sqrt{\hat{J}}}{4} \alpha^{3 / 2} .
$$

Observe that $\alpha$ is determined in terms of the fluxes $h_{A}$ and the $P^{A}$ through (4.2), and so it is independent of $J$ and $q_{A}$.

In deriving the above solution, we have assumed that all the $P^{A}$ are turned on so as to ensure the invertibility of the matrix $C_{A B}$. In this generic case, the constant values of the scalar fields $X^{A}$ and $A_{z}^{A}$ are entirely determined in terms of the $h_{A}, P^{A}$ and $q_{A}$. When switching off some of the $P^{A}$, some of the $A_{z}^{A}$ may be allowed to have arbitrary constant values, but these are expected not to contribute to the entropy density.

The $\mathrm{BTZ} \times \mathbb{R}^{2}$-solution given above can be found in any $N=2, \mathrm{U}(1)$ gauged supergravity model. This can also be inferred as follows. Setting $B^{\prime}=\left(X^{A}\right)^{\prime}=\left(A_{z}^{A}\right)^{\prime}=\hat{q}_{A}=0$ in the Lagrangian (2.18) and using the relations (4.3) and (4.4) yields a one-dimensional Lagrangian that descends from a three-dimensional Lagrangian describing Einstein gravity in the presence of an anti-de Sitter cosmological constant $\Lambda=-1 / l^{2}$ determined by the flux potential $\left(4 / l^{2}=\left(g h_{A} X^{A}\right)^{2}\right)$. As is well-known, the associated three-dimensional equations of motion allow for extremal BTZ black hole solutions with rotation. As shown in [19], the near-horizon geometry of the $\mathrm{BTZ} \times \mathbb{R}^{2}$-solution (which is supported by the magnetic fields (4.2)) preserves half of the supersymmetry.

The entropy of the BTZ black hole solution depends on $\hat{J}$, which takes the form

$$
J+\frac{1}{2} C^{A B} q_{A} q_{B}
$$

This combination is invariant under the transformation

$$
J \rightarrow \hat{J}, \quad q_{A} \rightarrow \hat{q}_{A}
$$

with $\hat{J}$ and $\hat{q}_{A}$ given in (2.17) and (2.12), respectively. In the absence of fluxes, this transformation is called spectral flow transformation and can be understood as follows from the supergravity perspective [26]. The rewriting of the five-dimensional Lagrangian in terms of first-order flow equations makes use of the combinations $\hat{q}_{A}$ and $\hat{J}$. These combinations have their origin in the presence of the gauge Chern-Simons term. When the $A_{z}^{A}$ are constant, the shifts $q_{A} \rightarrow \hat{q}_{A}$ and $J \rightarrow \hat{J}$ take the form of shifts induced by a large gauge transformation of $A_{z}^{A}$, i.e. $A^{A} \rightarrow A^{A}+k^{A}$, where $k^{A}$ denotes a closed one-form. These transformations constitute a symmetry of string theory, and this implies that the entropy of a black hole should be invariant under spectral flow. It must therefore depend on the combination (4.21). In the presence of fluxes, we find that the BTZ $\times \mathbb{R}^{2}$-solution (4.19) respects the spectral flow transformation (4.22).

The three-dimensional extremal BTZ black hole geometry, resulting from dimensionally reducing the black brane solution (4.19) on $\mathbb{R}^{2}$, is a state in the two-dimensional CFT dual to the asymptotic $A d S_{3}$, with left-moving central charge $c=\frac{3 l}{2 G_{3}}$ and $L_{0}-\frac{c}{24}=\frac{\rho_{+}^{2}}{4 G_{3} l}$, 
as well as $\tilde{L}_{0}-\frac{\tilde{c}}{24}=0$, cf. [33]. Hence, the large charge leading term in the entropy of the black hole is given by the Ramanujan-Hardy-Cardy formula for the dual CFT,

$$
\mathcal{S}_{\mathrm{BTZ}}=2 \pi \sqrt{\frac{c}{6}\left(L_{0}-\frac{c}{24}\right)} .
$$

This is exactly equal to the Bekenstein-Hawking entropy (4.20) computed above (in units of $G_{3}=1$ ), and can be regarded as a microscopic computation of the bulk black brane entropy from the holographic dual CFT.

4.1.2 Taking $P^{A}=0, q_{A}=0, \hat{J} \neq 0$

Now we consider the case when all the $P^{A}$ vanish. Then, (4.1) reduces to

$$
\left(h_{C} X^{C}\right) X_{A}=h_{A},
$$

which determines the constants $X^{A}$ in terms of the fluxes $h_{A}$. Contracting (4.24) with $G^{A B} h_{B}$ yields

$$
G^{A B} h_{A} h_{B}=\frac{2}{3}\left(h_{A} X^{A}\right)^{2} .
$$

Thus we take $h_{A} X^{A} \neq 0$ in the following, since otherwise $h_{A}=0$.

Combining the fourth equation of (2.21) with (2.23) results in

$$
B^{\prime}=\frac{1}{3} g h_{A} X^{A},
$$

which can be readily integrated to give

$$
e^{B}=e^{\beta} e^{\frac{1}{3} g h_{A} X^{A} r},
$$

where $\beta$ denotes an integration constant which we set to zero. The combination $U+W$ is given by

$$
U+W=2 B+u,
$$

where $u$ denotes an integration constant which we also set to zero.

The flow equation for $U-W$ reads

$$
\left(e^{-(U-W)}\right)^{\prime}=-\hat{J} e^{-4 B}=-\hat{J} e^{-\frac{4}{3} g h_{A} X^{A} r} .
$$

Next, let us consider the flow equation for $A_{z}^{A}$,

$$
\left(A_{z}^{A}\right)^{\prime}=-\frac{1}{2} G^{A C} q_{C} e^{-2 B} .
$$

A non-vanishing $q_{A}$ yields a running scalar field $A_{z}^{A} \sim G^{A B} q_{B} e^{-\frac{2}{3} g h_{A} X^{A} r}$. In the chosen coordinates, the line element can only have a throat at $|r|=\infty$. At either of these points, either the area element $e^{B}$ or $A_{z}^{A}$ blows up. If we demand that both $e^{B}$ and $A_{z}^{A}$ stay finite at the horizon, we are thus led to take $A_{z}^{A}$ to be constant, which can be obtained by setting $q_{A}=0$. Therefore, we set $q_{A}=0$ in the following. This implies that $\hat{J}$ is constant, which we take to be non-vanishing. 
Taking $h_{A} X^{A} \neq 0,(4.29)$ is solved by

$$
e^{-(U-W)}=\frac{3}{4} \frac{\hat{J}}{g h_{A} X^{A}} e^{-\frac{4}{3} g h_{A} X^{A} r}+\gamma,
$$

where $\gamma$ denotes an integration constant. Using (4.28), this results in

$$
\begin{aligned}
e^{2 W} & =\frac{3}{4} \frac{\hat{J}}{g h_{A} X^{A}} e^{-\frac{2}{3} g h_{A} X^{A} r}+\gamma e^{\frac{2}{3} g h_{A} X^{A} r}, \\
e^{-2 U} & =\frac{3}{4} \frac{\hat{J}}{g h_{A} X^{A}} e^{-2 g h_{A} X^{A} r}+\gamma e^{-\frac{2}{3} g h_{A} X^{A} r} .
\end{aligned}
$$

Redefining the radial coordinate,

$$
\tau=e^{\frac{1}{3} g h_{A} X^{A} r}, \quad \tau \geq 0
$$

yields

$$
\begin{aligned}
e^{B} & =\tau, \\
e^{2 W} & =\frac{3}{4} \frac{\hat{J}}{g h_{A} X^{A}} \tau^{-2}+\gamma \tau^{2}, \\
e^{-2 U} & =\frac{3}{4} \frac{\hat{J}}{g h_{A} X^{A}} \tau^{-6}+\gamma \tau^{-2}, \\
C & =\frac{\tau^{4}}{\frac{3}{4} \frac{\hat{J}}{g h_{A} X^{A}}+\gamma \tau^{4}}+\lambda .
\end{aligned}
$$

In the chosen coordinates, the line element reads

$$
d s^{2}=-e^{2 U(\tau)} d t^{2}+\left(\frac{1}{3} g h_{A} X^{A}\right)^{-2}\left(\frac{d \tau}{\tau}\right)^{2}+e^{2 B(\tau)}\left(d x^{2}+d y^{2}\right)+e^{2 W(\tau)}(d z+C(\tau) d t)^{2} .
$$

It exhibits a throat as $\tau \rightarrow 0$. In the following we set $\lambda=0$, and we take $\hat{J} /\left(g h_{A} X^{A}\right)>0$.

In the throat region, the terms proportional to $\gamma$ do not contribute (recall that we are taking $\hat{J}$ to be non-vanishing) and the line element becomes

$$
d s^{2}=-\tau^{6} d t^{2}+\left(\frac{1}{3} g h_{A} X^{A}\right)^{-2}\left(\frac{d \tau}{\tau}\right)^{2}+\tau^{2}\left(d x^{2}+d y^{2}\right)+\tau^{-2}\left(d z+\tau^{4} d t\right)^{2},
$$

where we rescaled the coordinates by various constant factors. Then, performing the coordinate transformation

$$
\tilde{\tau}=\tau^{3}, \tilde{t}=3 t,
$$

and setting $\left(\frac{1}{3} g h_{A} X^{A}\right)^{2}=1$ for convenience, the line element becomes

$$
\begin{aligned}
d s^{2} & =\frac{1}{9}\left(-\tilde{\tau}^{2} d \tilde{t}^{2}+\left(\frac{d \tilde{\tau}}{\tilde{\tau}}\right)^{2}\right)+\tilde{\tau}^{2 / 3}\left(d x^{2}+d y^{2}\right)+\tilde{\tau}^{-2 / 3}\left(d z+\frac{1}{3} \tilde{\tau}^{4 / 3} d \tilde{t}\right)^{2} \\
& =\frac{2}{3} \tilde{\tau}^{2 / 3} d \tilde{t} d z+\tilde{\tau}^{-2 / 3} d z^{2}+\frac{1}{9}\left(\frac{d \tilde{\tau}}{\tilde{\tau}}\right)^{2}+\tilde{\tau}^{2 / 3}\left(d x^{2}+d y^{2}\right)
\end{aligned}
$$

which describes a null-warped throat. The entropy density vanishes, $\left.\mathcal{S} \sim e^{2 B+W}\right|_{\tau=0}=0$. 
In the limit $\tau \rightarrow \infty$, on the other hand, there are two distinct cases. When $\gamma \neq 0$ (we take $\gamma>0)$,

$$
\begin{aligned}
e^{B} & =\tau, \\
e^{2 W} & \approx \gamma \tau^{2}, \\
e^{-2 U} & \approx \gamma \tau^{-2}, \\
C & \approx \gamma^{-1},
\end{aligned}
$$

and the line element becomes

$$
d s^{2}=-\tau^{2} d t^{2}+\left(\frac{1}{3} g h_{A} X^{A}\right)^{-2}\left(\frac{d \tau}{\tau}\right)^{2}+\tau^{2}\left(d x^{2}+d y^{2}\right)+\tau^{2}(d z+d t)^{2},
$$

where we rescaled the coordinates. Observe that this describes a patch of $A d S_{5}$.

The other case corresponds to setting $\gamma=0$, in which case the behavior at $\tau \rightarrow \infty$ is determined by

$$
\begin{aligned}
e^{B} & =\tau, \\
e^{2 W} & =\frac{3}{4} \frac{\hat{J}}{g h_{A} X^{A}} \tau^{-2}, \\
e^{-2 U} & =\frac{3}{4} \frac{\hat{J}}{g h_{A} X^{A}} \tau^{-6}, \\
C & =\frac{4}{3} \frac{g h_{A} X^{A}}{\hat{J}} \tau^{4},
\end{aligned}
$$

and the associated line element is again of the form (4.36) and (4.38).

Thus, we conclude that the solution (4.35) with $\gamma \neq 0$ describes a solution that interpolates between $A d S_{5}$ and a null-warped Nernst throat at the horizon with vanishing entropy, in which all the scalar fields are kept constant. This is a purely gravitational stationary solution that is supported by electric fluxes $h_{A}$. It is an example of a Nernst brane (i.e. a solution with vanishing entropy density), and is the five-dimensional counterpart of the four-dimensional Nernst solution constructed in [6]. Nernst solutions suffer from the problem of divergent tidal forces. These may get cured by quantum or stringy effects [34].

\subsection{Solutions with non-constant scalar fields $X^{A}$}

Here we present numerical solutions that are supported by non-constant scalar fields $X^{A}$ and that interpolate between a near horizon solution of the type discussed above in section 4.1.1 and an asymptotic $A d S_{5}$-region with metric

$$
d s^{2}=-e^{2 r} d t^{2}+d r^{2}+e^{2 r}\left(d x^{2}+d y^{2}+d z^{2}\right),
$$

i.e. the metric functions $U(r), B(r)$ and $W(r)$ in (2.1) all asymptote to the linear function $r$ and $C$ becomes 0 (or constant, since a constant $C$ can be removed by a redefinition of the $z$-variable). To be concrete, we work within the STU-model. Within this model, a solution with a single running scalar and with $\hat{J}=0$ was already given in section 2.3 of [7]. 
In order to facilitate the comparison with their results, we will work with physical scalars in this section, i.e. we solve the constraint $X^{1} X^{2} X^{3}=1$ via

$$
X^{1}=e^{-\frac{1}{\sqrt{6}} \phi^{1}-\frac{1}{\sqrt{2}} \phi^{2}}, \quad X^{2}=e^{-\frac{1}{\sqrt{6}} \phi^{1}+\frac{1}{\sqrt{2}} \phi^{2}}, \quad X^{3}=e^{\frac{2}{\sqrt{6}} \phi^{1}} .
$$

In an asymptotically $A d S_{5}$-spacetime the two scalars $\phi^{i}$ have a leading order expansion

$$
\phi^{i}=a_{i} r e^{-2 r}+b_{i} e^{-2 r}+\mathcal{O}\left(e^{-4 r}\right), \quad i=1,2,
$$

i.e. they both correspond to dimension 2 operators of the dual field theory and $a_{i}$ and $b_{i}$ correspond to the sources and 1-point functions, respectively.

For all the following numerical solutions, we choose

$$
g=1, h_{1}=h_{2}=h_{3}=1 .
$$

\subsubsection{Solution with a single running scalar}

Let us first consider the case with a single running scalar field and with vanishing $\hat{J}$. For concreteness, we choose the magnetic fields

$$
P^{1}=P^{2}=4^{1 / 3}, \quad P^{3}=-2 \cdot 4^{1 / 3},
$$

so as to satisfy the constraint (2.22). One could choose a different overall normalization for the magnetic fields $P^{A}$ by rescaling the $x$ and $y$ coordinates. This, on the other hand, would also imply a rescaling of $e^{B}$ and, thus, we would not have $B=0$ anymore, as was assumed in section 4.1.1. Solving (4.2) for these values of $P^{A}$ leads to

$$
X_{(0)}^{1}=X_{(0)}^{2}=4^{-1 / 3}, \quad X_{(0)}^{3}=4^{2 / 3},
$$

where we added the subscript (0) in order to distinguish the constant near horizon values from the full, non-constant solution to be discussed momentarily. These values lead to

$$
\phi_{(0)}^{1} \approx 1.132, \quad \phi_{(0)}^{2}=0, \quad \alpha=3 \cdot 2^{1 / 3} \approx 3.780,
$$

with $\alpha$ defined in (4.13). Finally, using (4.12), (4.14) and (4.16), we obtain

$$
U_{(0)}=-\frac{1}{2} \ln \left(4 \alpha^{-3}\right)+\frac{\alpha}{2} r, \quad W_{(0)}=\frac{1}{2} \ln \left(4 \alpha^{-3}\right)+\frac{\alpha}{2} r, \quad C_{(0)}=0 .
$$

In order to obtain an interpolating solution with an asymptotic $A d S_{5}$-region, we slightly perturb around this solution. In particuar, we make the following ansatz for small $r$

$$
\begin{aligned}
X^{1} & =X_{(0)}^{1}+c_{1} e^{c_{2} r}, & X^{2} & =X_{(0)}^{2}+c_{3} e^{c_{4} r}, \\
B & =\delta B, & U & =U_{(0)}+\delta U,
\end{aligned} \quad W=W_{(0)}+\delta W
$$

with $c_{2}, c_{4}>0$. Plugging this into the flow equations (2.21), we obtain the following conditions:

$$
\begin{aligned}
c_{1} & =c_{3}, & c_{2} & =c_{4}=2^{-2 / 3}(\sqrt{33}-1) \approx 2.989, \\
\delta U & =\delta W=-\frac{1}{2} \frac{c_{3}\left(2^{2 / 3} c_{2}+14\right)}{2} e^{c_{2} r}, & \delta B & =2^{-2 / 3} c_{3}\left(3 \cdot 2^{1 / 3}+c_{2}\right) e^{c_{2} r}
\end{aligned}
$$



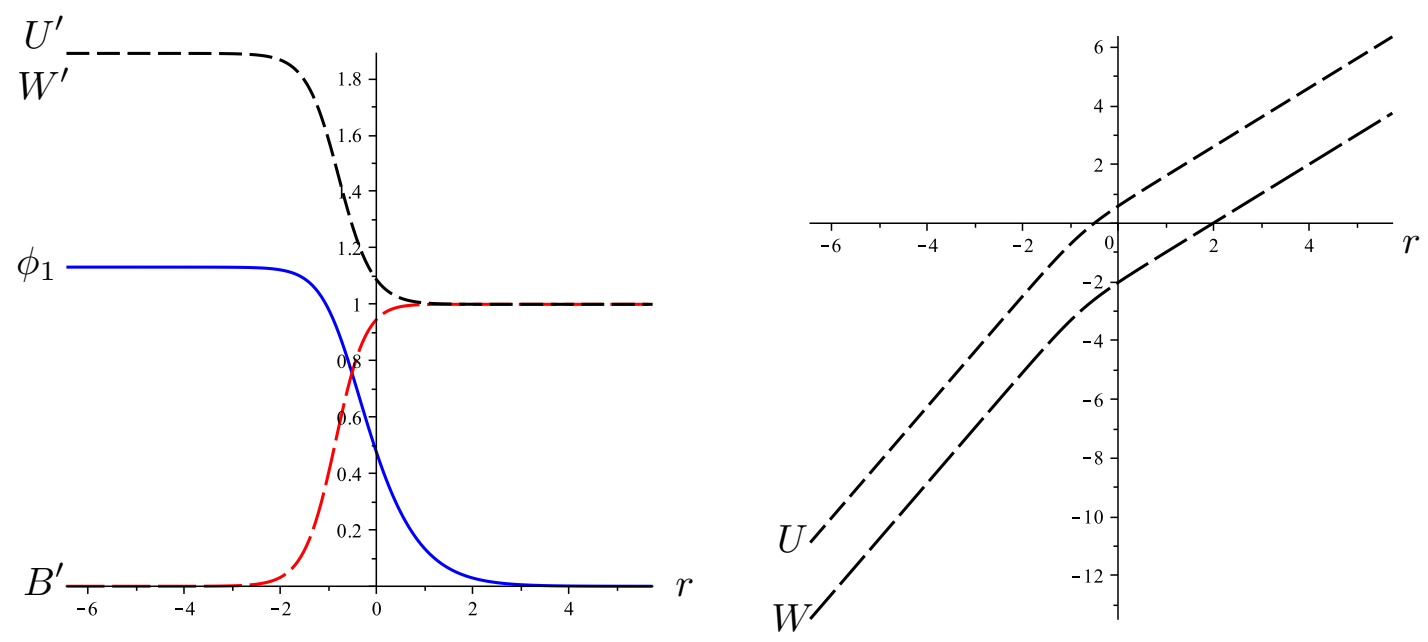

Figure 1. The case of one scalar with vanishing $\hat{J}$.

The constant $c_{3}$ is undetermined and sets the value of the source and 1-point function of $\phi^{1}$, cf. (4.44). We will see this explicitly in the example in section 4.2.3 below. Using (4.51), we can find the initial conditions needed to solve (2.21) numerically. In practice it is most convenient to solve (2.21) in the $\tau$ variable (4.12), as the horizon is at $\tau=0$. This allows to set the initial conditions for instance at $\tau=10^{-13}$ and then integrate outwards. Doing so and choosing $c_{3}=1$, we obtain the result depicted in figure 1 (note that the plot makes use of the $r$-variable, i.e. the primes denote derivatives with respect to $r$ as before). Moreover, $C \equiv 0$. Even though the functions $U$ and $W$ always have the same derivative, they differ by a shift, as can be seen in the right part of figure 1 . This is due to the fact that we chose $b=4 \alpha^{-3}$ according to (4.16), instead of $b=1$, with $b$ being introduced in (4.9). This solution is very similar to the one discussed in section 2.3 of [7] and it is obvious that the metric asymptotically becomes of the form (4.42).

\subsubsection{Solution with two running scalars, $\hat{J}=0$}

We now turn to the case of two non-trivial scalars, first still with vanishing $\hat{J}$ and then with $\hat{J} \neq 0$ in the next subsection. In all cases we choose

$$
P^{1}=\left(\frac{4}{3}\right)^{1 / 3}, \quad P^{2}=2 \cdot\left(\frac{4}{3}\right)^{1 / 3}, \quad P^{3}=-3 \cdot\left(\frac{4}{3}\right)^{1 / 3}
$$

Again, the overall normalization of the $P^{A}$ is imposed on us by demanding $B=0$ in the near-horizon region. This time solving (4.2) leads to

$$
X_{(0)}^{1}=\frac{1}{4}\left(\frac{4}{3}\right)^{2 / 3}, \quad X_{(0)}^{2}=\left(\frac{4}{3}\right)^{2 / 3}, \quad X_{(0)}^{3}=\frac{3}{2} 6^{1 / 3},
$$

which implies

$$
\phi_{(0)}^{1} \approx 1.228, \quad \phi_{(0)}^{2} \approx 0.980, \quad \alpha=\frac{7}{6} \cdot 4^{2 / 3} \cdot 3^{1 / 3} \approx 4.240 .
$$




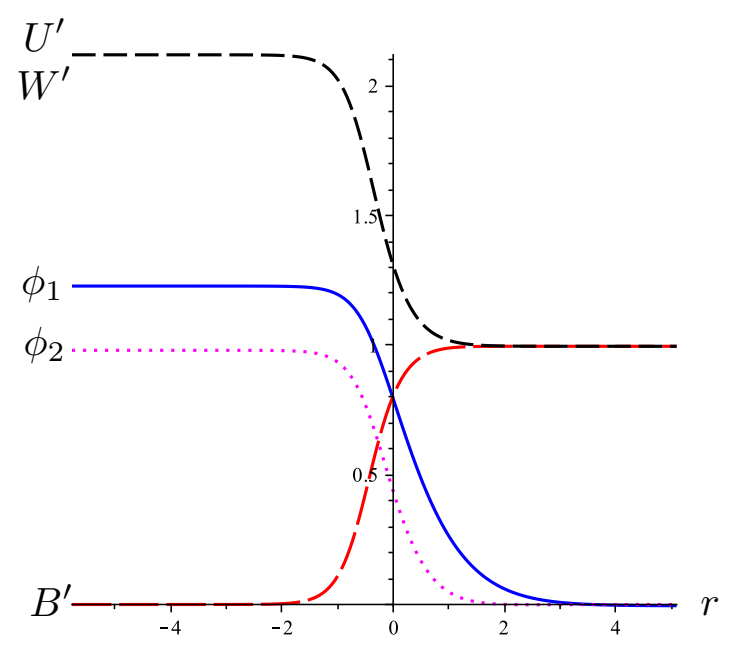

Figure 2. The case of two scalars with vanishing $\hat{J}$.

The functions $U_{(0)}, W_{(0)}$ and $C_{(0)}$ are again given by (4.49), now using the value of $\alpha$ given in (4.54).

Perturbing around the near-horizon solution utilizes the same ansatz as in (4.50). This time, we obtain the conditions

$$
\begin{aligned}
c_{1} & =-\frac{c_{3}}{8} \frac{41 \cdot 6^{1 / 3}+33 c_{2}}{29 \cdot 6^{1 / 3}-3 c_{2}}, \quad c_{2}=c_{4} \approx 3.694, \\
\delta U & =\delta W=\frac{1}{16} \frac{c_{3}\left(21 \cdot 6^{2 / 3} c_{2}^{2}+588 c_{2}-178 \cdot 6^{1 / 3}\right)}{c_{2}\left(-3 c_{2}+29 \cdot 6^{1 / 3}\right)} e^{c_{2} r} \\
\delta B & =\frac{6^{1 / 3}}{16} \frac{c_{3}\left(-27 c_{2}^{2}-42 \cdot 6^{1 / 3} c_{2}+49 \cdot 6^{2 / 3}\right)}{-3 c_{2}+29 \cdot 6^{1 / 3}} e^{c_{2} r} .
\end{aligned}
$$

Again, the parameter $c_{3}$ determines the sources and 1-point functions of the scalars $\phi^{1}$ and $\phi^{2}$.

Using (4.55) in (4.50) we obtain the initial conditions to solve the flow equations numerically. We do not find any solution for $c_{3}=1$, but inverting the sign, i.e. choosing $c_{3}=-1$, leads to the solution depicted in figure 2 , which in addition has $C \equiv 0$ and which is asymptotically $A d S_{5}$.

\subsubsection{Solutions with two running scalars, $\hat{J} \neq 0$}

Finally, let us look at the more general case, where we have two running scalars and a constant non-vanishing $\hat{J}$. We choose the same values for the magnetic fields as in the last example, i.e. (4.52). Given that (4.2) does not depend on $\hat{J}$ at all, it is not surprising that this leads to the same values for the $X_{(0)}^{A}$ as in (4.53) (and, thus, also (4.54) does not change). The main change arises for $U, W$ and $C$, as their flow equations explicitly depend 

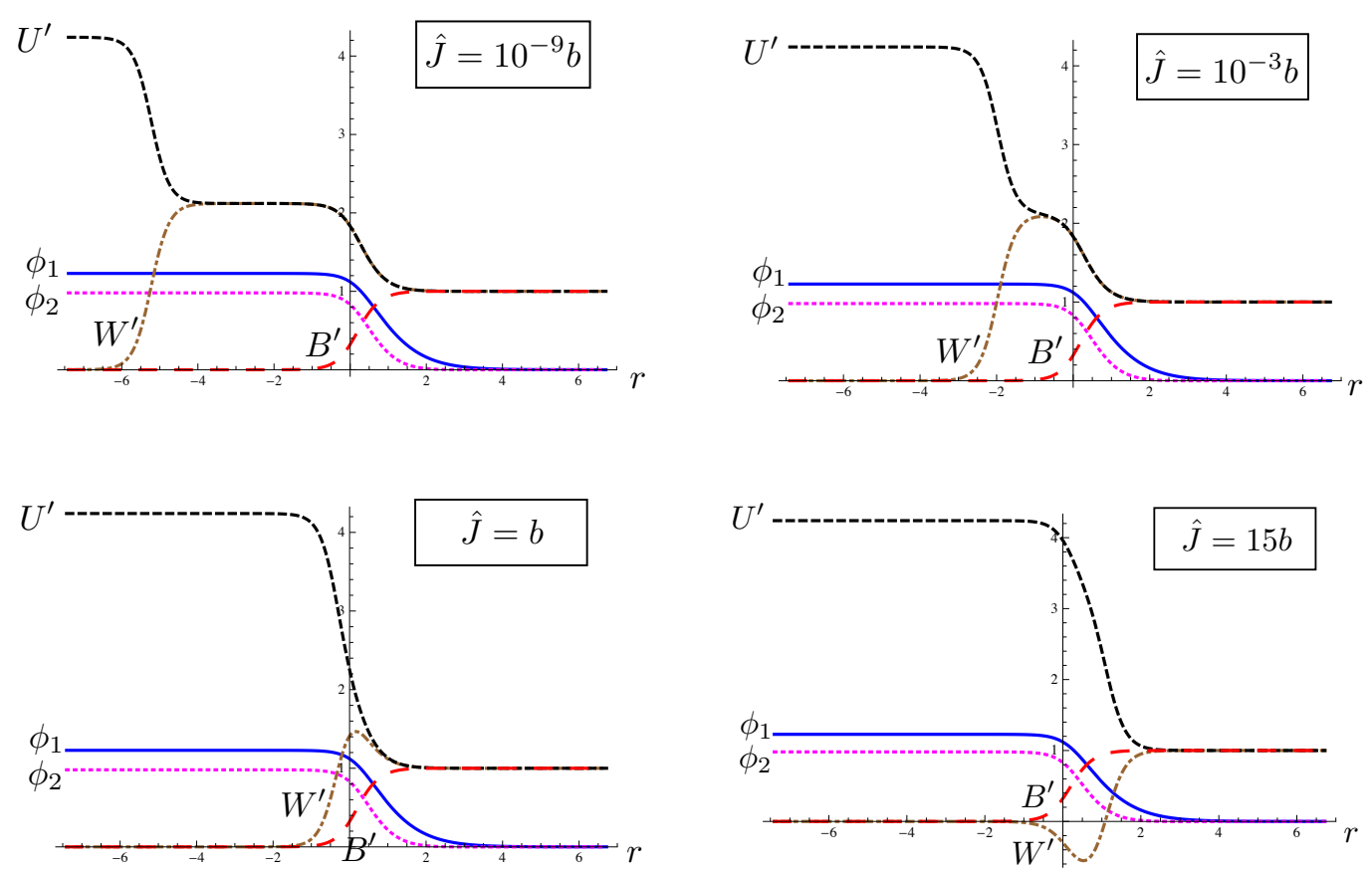

Figure 3. The case of two scalars witht non-vanishing $\hat{J}$.

on $\hat{J}$. They take on the near-horizon form

$$
\begin{aligned}
U_{(0)} & =-\frac{1}{2} \ln \left(\frac{\hat{J}}{\alpha}+\frac{4 e^{\alpha r}}{\alpha^{3}}\right)+\alpha r, \quad W_{(0)}=\frac{1}{2} \ln \left(\frac{\hat{J}}{\alpha}+\frac{4 e^{\alpha r}}{\alpha^{3}}\right), \\
C_{(0)} & =\frac{\alpha^{3}}{4}\left(\frac{1}{\hat{J} \frac{\alpha^{2}}{4} e^{-\alpha r}+1}-1\right) .
\end{aligned}
$$

Notice, in particular, the different behavior of $U_{(0)}$ and $W_{(0)}$ very close to the horizon, i.e. for $r \rightarrow-\infty$. Whereas the slope of $U_{(0)}$ and $W_{(0)}$ was $\alpha / 2$ in the case of vanishing $\hat{J}$, cf. (4.49), now it is $\alpha$ for $U_{(0)}$ and zero for $W_{(0)}$ in the case of non-vanishing $\hat{J}$. We will clearly see this in the numerical solutions.

Again, we perturb around the near-horizon solution by (4.50). We again infer that $c_{2}=c_{4}$ and that $c_{1}$ and $c_{3}$ are related as in (4.55). Moreover, $\delta U, \delta W$ and $\delta B$ are all proportional to $e^{c_{2} r}$. Without going into the details, we present the resulting numerical solutions for different values of $\hat{J}$ in figure 3 . All these plots were produced using $c_{3}=-0.1$ and $b=4 \alpha^{-3}$. One can nicely see that the main difference to the case of vanishing $\hat{J}$ appears in the $U$ and $W$ sector. The different slope of $U$ and $W$ close to the horizon, mentioned in the last paragraph, is apparent. It is also obvious that for small $\hat{J}, U$ and $W$ first behave as in the case with vanishing $\hat{J}$ when approaching the horizon from infinity. I.e. they start out showing the same slope of $\alpha / 2$ until the $\hat{J}$-term starts dominating very close to the horizon, where the slope of $U$ doubles and $W$ becomes constant. With increasing $\hat{J}$ the intermediate region, where $U$ and $W$ have the same slope of $\alpha / 2$, becomes smaller and smaller and finally disappears altogether. 


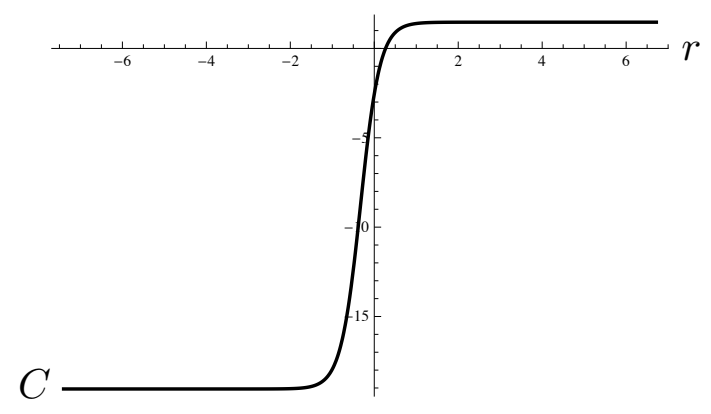

Figure 4. The function $C$ for $\hat{J}=b$.
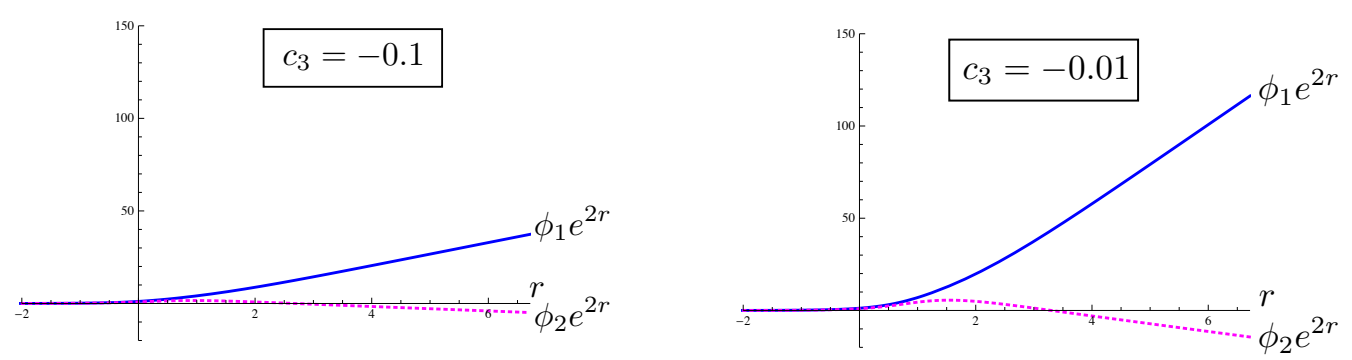

Figure 5. The two scalars multiplied with $e^{2 r}$.

The function $C=e^{U-W}-1 / b$ is shown (for $\hat{J}=b$ ) in figure 4. Obviously, asymptotically it becomes constant and, thus, the asymptotic region is indeed given by $A d S_{5}$.

Finally, in figure 5, we plot $\phi^{1}$ and $\phi^{2}$, multiplied with $e^{2 r}$, for two different values of $c_{3}$. As expected from (4.44), the graphs show a linear behavior with non-vanishing sources and 1-point functions for the two operators dual to the scalars. Obviously, these sources and 1-point functions depend on the value of $c_{3}$.

\section{Acknowledgments}

We acknowledge helpful discussions with Costas Bachas, Gianguido Dall'Agata, Oscar Dias, Pau Figueras, Vishnu Jejjala, Amir-Kian Kashani-Poor, Niels Obers, Giuseppe Policastro, Harvey Reall and Jan Troost. The work of G.L.C. was partially supported by the Center for Mathematical Analysis, Geometry and Dynamical Systems (IST/Portugal), as well as by Fundação para a Ciência e a Tecnologia (FCT/Portugal) through grants CERN/FP/116386/2010 and PTDC/MAT/119689/2010. The work of S.B., M.H. and S.N. was supported by the Excellence Cluster "The Origin and the Structure of the Universe" in Munich. The work of M.H. and S.N. was also supported by the German Research Foundation (DFG) within the Emmy-Noether-Program (grant number: HA 3448/3-1). The work of S.B., G.L.C., M.H. and S.N. was supported in part by the transnational cooperation FCT/DAAD grant "Black Holes, duality and string theory". 


\section{A Einstein equations}

When evaluated on the solution ansatz (2.1), the independent Einstein equations take the following form:

$t$-component:

$$
\begin{aligned}
\frac{1}{2} e^{-2(U+V)}\left(-e^{2(U+W)} C^{2}-2 e^{2(U+W)} C\left(2 B^{\prime} C^{\prime}-C^{\prime}\left(U^{\prime}+V^{\prime}-3 W^{\prime}\right)+C^{\prime \prime}\right)\right. \\
\quad+2 e^{4 U}\left(2 B^{\prime} U^{\prime}+U^{\prime 2}-U^{\prime} V^{\prime}+U^{\prime} W^{\prime}+U^{\prime \prime}\right) \\
\left.\quad-e^{2 W} C^{2}\left(e^{2 W} C^{\prime 2}+2 e^{2 U}\left(2 B^{\prime} W^{\prime}+U^{\prime} W^{\prime}-V^{\prime} W^{\prime}+W^{\prime 2}+W^{\prime \prime}\right)\right)\right) \\
=\left(-e^{2 U}+e^{2 W} C^{2}\right)\left(\frac{1}{3} g^{2}\left(G^{A B} h_{A} h_{B}-2\left(h_{A} X^{A}\right)^{2}\right)-\frac{1}{6} G_{A B} F_{K L}^{A} F^{B K L}\right)+G_{A B} F_{r t}^{A} F_{r t}^{B} e^{-2 V}
\end{aligned}
$$

rr-component:

$$
\begin{gathered}
-2 B^{\prime 2}+\frac{1}{2} e^{2 W-2 U} C^{2}-U^{\prime 2}+2 B^{\prime} V^{\prime}+U^{\prime} V^{\prime}+V^{\prime} W^{\prime}-W^{\prime 2}-2 B^{\prime \prime}-U^{\prime \prime}-W^{\prime \prime} \\
=G_{A B}\left(X^{A}\right)^{\prime}\left(X^{B}\right)^{\prime}+e^{2 V}\left(\frac{1}{3} g^{2}\left(G^{A B} h_{A} h_{B}-2\left(h_{A} X^{A}\right)^{2}\right)-\frac{1}{6} G_{A B} F_{K L}^{A} F^{B K L}\right) \\
+G_{A B}\left(F_{r t}^{A} F_{r t}^{B}\left(-e^{-2 U}\right)+\left(A_{z}^{A}\right)^{\prime}\left(A_{z}^{B}\right)^{\prime}\left(e^{-2 W}-e^{-2 U} C^{2}\right)+2 F_{r t}^{A}\left(A_{z}^{B}\right)^{\prime} e^{-2 U} C\right)
\end{gathered}
$$

$x x$-component:

$$
\begin{aligned}
& -e^{2 B-2 V}\left(2 B^{\prime 2}+B^{\prime} U^{\prime}-B^{\prime} V^{\prime}+B^{\prime} W^{\prime}+B^{\prime \prime}\right)= \\
& e^{2 B}\left(\frac{1}{3} g^{2}\left(G^{A B} h_{A} h_{B}-2\left(h_{A} X^{A}\right)^{2}\right)-\frac{1}{6} G_{A B} F_{K L}^{A} F^{B K L}\right)+G_{A B} P^{A} P^{B} e^{-2 B}
\end{aligned}
$$

$z z$-component:

$$
\begin{aligned}
& \frac{1}{2} e^{2 W-2 U-2 V}\left(-e^{2 W} C^{\prime 2}-2 e^{2 U}\left(2 B^{\prime} W^{\prime}+U^{\prime} W^{\prime}-W^{\prime} V^{\prime}+W^{\prime 2}+W^{\prime \prime}\right)\right) \\
& =e^{2 W}\left(\frac{1}{3} g^{2}\left(G^{A B} h_{A} h_{B}-2\left(h_{A} X^{A}\right)^{2}\right)-\frac{1}{6} G_{A B} F_{K L}^{A} F^{B K L}\right)+G_{A B}\left(A_{z}^{A}\right)^{\prime}\left(A_{z}^{B}\right)^{\prime} e^{-2 V}
\end{aligned}
$$

$t z$-component:

$$
\begin{aligned}
& \frac{1}{2} e^{2 W-2 U-2 V}\left(-2 e^{2 U}\left(B^{\prime} C^{\prime}+2 C B^{\prime} W^{\prime}\right)+e^{2 U}\left(C^{\prime} U^{\prime}+C^{\prime} V^{\prime}-3 C^{\prime} W^{\prime}-C^{\prime \prime}\right)-e^{2 W} C C^{\prime 2}\right. \\
& \left.\quad+2 e^{2 U} C\left(U^{\prime} W^{\prime}-V^{\prime} W^{\prime}+W^{\prime 2}+W^{\prime \prime}\right)\right) \\
& =e^{2 W} C\left(\frac{1}{3} g^{2}\left(G^{A B} h_{A} h_{B}-2\left(h_{A} X^{A}\right)^{2}\right)-\frac{1}{6} G_{A B} F_{K L}^{A} F^{B K L}\right)+2 G_{A B} F_{r t}^{A}\left(A_{z}^{B}\right)^{\prime} e^{-2 V}
\end{aligned}
$$

\section{B Relating five- and four-dimensional flow equations}

We relate the four-dimensional flow equations for black branes derived in [6] in big moduli space to the five-dimensional flow equations (2.21). We set $g=1$ throughout. 
The four-dimensional $N=2, \mathrm{U}(1)$ gauged supergravity theory is based on complex scalar fields $X^{I}$ encoded in the cubic prepotential (with $A=1, \ldots, n$ )

$$
F(X)=-\frac{1}{6} \frac{C_{A B C} X^{A} X^{B} X^{C}}{X^{0}}=\left(X^{0}\right)^{2} \mathcal{F}(z),
$$

where $z^{A}=X^{A} / X^{0}$ denote the physical scalar fields and

$$
\mathcal{F}(z)=-\frac{1}{6} C_{A B C} z^{A} z^{B} z^{C}
$$

Differentiating with respect to $z^{A}$ yields

$$
\begin{aligned}
\mathcal{F}_{A} & =-\frac{1}{2} C_{A B C} z^{B} z^{C}, \\
\mathcal{F}_{A B} & =-C_{A B C} z^{C}
\end{aligned}
$$

where $\mathcal{F}_{A}=\partial \mathcal{F} / \partial z^{A}$, etc. The Kähler potential $K(z, \bar{z})$ is determined in terms of $\mathcal{F}$ by

$$
\begin{aligned}
e^{-K} & =i\left(2(\mathcal{F}-\overline{\mathcal{F}})-\left(z^{A}-\bar{z}^{A}\right)\left(\mathcal{F}_{A}+\overline{\mathcal{F}}_{A}\right)\right) \\
& =\frac{i}{6} C_{A B C}\left(z^{A}-\bar{z}^{A}\right)\left(z^{B}-\bar{z}^{B}\right)\left(z^{C}-\bar{z}^{C}\right) .
\end{aligned}
$$

The Kähler metric $g_{A \bar{B}}=\partial_{A} \partial_{\bar{B}} K(z, \bar{z})$ can be expressed as

$$
g_{A \bar{B}}=K_{A} K_{\bar{B}}-i e^{K}\left(\mathcal{F}_{A B}-\overline{\mathcal{F}}_{A B}\right)
$$

where $K_{A}=\partial K / \partial z^{A}$ and

$$
K_{A}=-K_{\bar{A}}=-e^{K} \frac{i}{2} C_{A B C}\left(z^{B}-\bar{z}^{B}\right)\left(z^{C}-\bar{z}^{C}\right) .
$$

In the following we pick the gauge $X^{0}(z)=1, X^{A}(z)=z^{A}$ (with $X^{I}(z) \equiv X^{I} e^{-K / 2}$ ), so that the complex scalar fields $X^{I}$ and the $z^{A}$ are related by $X^{0}=e^{K / 2}$ and $X^{A}=e^{K / 2} z^{A}$.

Using the dictionary (3.5) that relates the quantities appearing in the four- and fivedimensional flow equations, in particular $z^{A}-\bar{z}^{A}=2 i e^{W} X_{5 \mathrm{~d}}^{A}$, we obtain

$$
e^{-K}=8 v e^{3 W}
$$

as well as

$$
\begin{aligned}
K_{\bar{A}} & =-\frac{3 i}{2 v} e^{-W} X_{A}^{5 \mathrm{~d}} \\
g_{A \bar{B}} & =4 v e^{K+W} G_{A B}=\frac{1}{2} e^{-2 W} G_{A B}
\end{aligned}
$$

where $G_{A B}$ denotes the target space metric in five dimensions, cf. (3.4), and the $X_{A}^{5 \mathrm{~d}}$ were defined in (3.3). The factor of $v$ in (B.7) arises due to the normalization in (3.2). Using these expressions, we establish

$$
g^{A \bar{B}} K_{\bar{B}}=-\left(z^{A}-\bar{z}^{A}\right) .
$$


In the big moduli space, the four-dimensional flow equations were expressed in terms of rescaled complex scalar fields $Y^{I}$ given by $Y^{0}=\left|Y^{0}\right| e^{i \alpha}$ and $Y^{A}=Y^{0} z^{A}$, where $\left|Y^{0}\right|=$ $e^{K / 2+\psi-U_{4}}$. On a solution to the four-dimensional flow equations we can relate the phase $\alpha$ to the phase $\gamma$ that enters in the four-dimensional flow equations. We obtain $\alpha=-\gamma$, which we establish as follows. Writing $e^{2 i \alpha}=Y^{0} / \bar{Y}^{0}$ we get

$$
\alpha^{\prime}=-\frac{i}{2} e^{-2 i \alpha}\left(\frac{\left(Y^{0}\right)^{\prime}}{\bar{Y}^{0}}-\left(\bar{Y}^{0}\right)^{\prime} \frac{Y^{0}}{\left(\bar{Y}^{0}\right)^{2}}\right)=-\frac{i}{2 \bar{Y}^{0}}\left(e^{-2 i \alpha}\left(Y^{0}\right)^{\prime}-\left(\bar{Y}^{0}\right)^{\prime}\right)
$$

The flow equation for $Y^{0}$ reads [6]

$$
\begin{aligned}
\left(Y^{0}\right)^{\prime} & =e^{\psi-U_{4}} N^{0 J} \bar{q}_{J} \\
& =e^{\psi-U_{4}+K}\left[g^{A \bar{B}} K_{A} \bar{q}_{\bar{J}}\left(\partial_{\bar{B}}+K_{\bar{B}}\right) \bar{X}^{\bar{J}}(\bar{z})-\bar{q}_{\bar{J}} \bar{X}^{\bar{J}}(\bar{z})\right],
\end{aligned}
$$

where we used

$$
N^{I J}=e^{K}\left[g^{A \bar{B}}\left(\partial_{A}+\partial_{A} K\right) X^{I}(z)\left(\partial_{\bar{B}}+\partial_{\bar{B}} K\right) \bar{X}^{J}(\bar{z})-X^{I}(z) \bar{X}^{J}(\bar{z})\right],
$$

cf. for instance [35]. In (B.11) the $q_{I}$ denote the four-dimensional quantities

$$
q_{I}=e^{U_{4}-2 \psi+i \gamma}\left(\hat{Q}_{I}-i e^{2\left(\psi-U_{4}\right)} \hat{h}_{I}\right)
$$

which should not be confused with the five-dimensional electric charges $q_{A}^{5 \mathrm{~d}}$. The quantities $\hat{Q}_{I}$ and $\hat{h}_{I}$ are combinations of the four-dimensional charges and fluxes given by [6]

$$
\begin{aligned}
& \hat{Q}_{I}=Q_{I}-F_{I J} P^{J} \\
& \hat{h}_{I}=h_{I}-F_{I J} h^{J} .
\end{aligned}
$$

For later use, we also introduce the quantities

$$
\begin{aligned}
Z(Y) & =-\hat{Q}_{I} Y^{I}, \\
W(Y) & =-\hat{h}_{I} Y^{I} .
\end{aligned}
$$

Inserting the flow equation (B.11) in (B.10) yields

$$
\begin{aligned}
\alpha^{\prime}=-\frac{i}{2} e^{i \alpha} e^{K / 2}[ & \left(g^{A \bar{B}} K_{A} K_{\bar{B}}-1\right)\left(e^{-2 i \alpha}\left(\bar{q}_{0}+\bar{q}_{\bar{C}} \bar{z}^{\bar{C}}\right)-\left(q_{0}+q_{C} z^{C}\right)\right) \\
& \left.+g^{A \bar{B}}\left(e^{-2 i \alpha} K_{A} \bar{q}_{\bar{B}}-q_{A} K_{\bar{B}}\right)\right]
\end{aligned}
$$

where we used the relation

$$
\bar{q}_{J}\left(\partial_{\bar{B}}+K_{\bar{B}}\right) \bar{X}^{J}(z)=\bar{q}_{\bar{B}}+K_{\bar{B}}\left(\bar{q}_{0}+\bar{q}_{A} \bar{z}^{A}\right)
$$

as well as $\left|Y^{0}\right|=e^{K / 2+\psi-U_{4}}$. Next, using that on a four-dimensional solution we have $q_{I} Y^{I}=\bar{q}_{I} \bar{Y}^{\bar{I}}$, we obtain

$$
e^{-2 i \alpha}\left(\bar{q}_{0}+\bar{q}_{\bar{C}} \bar{z}^{\bar{C}}\right)=q_{0}+q_{C} z^{C} .
$$


Inserting this in (B.16) results in

$$
\alpha^{\prime}=-\frac{i}{2} e^{i \alpha} e^{K / 2} g^{A \bar{B}}\left(e^{-2 i \alpha} K_{A} \bar{q}_{\bar{B}}-q_{A} K_{\bar{B}}\right)
$$

Using (B.9), we obtain

$$
\begin{aligned}
g^{A \bar{B}}\left(e^{-2 i \alpha} K_{A} \bar{q}_{\bar{B}}-q_{A} K_{\bar{B}}\right) & =\left(z^{A}-\bar{z}^{A}\right)\left(e^{-2 i \alpha} \bar{q}_{\bar{A}}+q_{A}\right) \\
& =e^{-2 i \alpha} z^{A} \bar{q}_{\bar{A}}-\bar{z}^{A} q_{A}-e^{-2 i \alpha} \bar{z}^{A} \bar{q}_{\bar{A}}+q_{A} z^{A} \\
& =e^{-2 i \alpha}\left(\bar{q}_{0}+z^{A} \bar{q}_{\bar{A}}\right)-\left(q_{0}+\bar{z}^{A} q_{A}\right),
\end{aligned}
$$

where we used (B.18) in the last equality. Now we notice that

$$
q_{I} \bar{z}^{I}=q_{0}+\bar{z}^{A} q_{A}=-e^{i(\alpha+\gamma)} e^{-K / 2+2 U_{4}-3 \psi}\left(\bar{Z}(\bar{Y})-i e^{2\left(\psi-U_{4}\right)} \bar{W}(\bar{Y})\right)
$$

and

$$
e^{-2 i \alpha} \bar{q}_{\bar{I}} z^{I}=e^{-2 i \alpha}\left(\bar{q}_{0}+z^{A} \bar{q}_{\bar{A}}\right)=-e^{-i(3 \alpha+\gamma)} e^{-K / 2+2 U_{4}-3 \psi}\left(Z(Y)+i e^{2\left(\psi-U_{4}\right)} W(Y)\right),
$$

so that

$$
\alpha^{\prime}=-e^{2 U_{4}-3 \psi} \operatorname{Im}\left(e^{-i(2 \alpha+\gamma)} Z(Y)\right)-e^{-\psi} \operatorname{Re}\left(e^{-i(2 \alpha+\gamma)} W(Y)\right) .
$$

This is precisely the flow equation for $\gamma$, provided $\alpha=-\gamma$.

Using this result, we now relate the flow equations for the $z^{A}$ to the five-dimensional flow equations for $X_{5 \mathrm{~d}}^{A}$ and $A_{z}^{A}$. Using the four-dimensional flow equations for $Y^{0}$ and $Y^{A}$ we obtain

$$
\begin{aligned}
\left(z^{A}\right)^{\prime} & =\frac{1}{Y^{0}}\left(\left(Y^{A}\right)^{\prime}-z^{A}\left(Y^{0}\right)^{\prime}\right) \\
& =\frac{e^{\psi-U_{4}}}{Y^{0}}\left(N^{A J}-z^{A} N^{0 J}\right) \bar{q}_{J} \\
& =e^{-\frac{K}{2}+i \gamma}\left(N^{A J}-z^{A} N^{0 J}\right) \bar{q}_{J} .
\end{aligned}
$$

Then, using (B.12), one derives

$$
N^{A J}-z^{A} N^{0 J}=e^{K} g^{A \bar{B}}\left(\partial_{\bar{B}}+K_{\bar{B}}\right) \bar{X}^{J}(z),
$$

which implies

$$
\left(z^{A}\right)^{\prime}=e^{\frac{K}{2}+i \gamma} g^{A \bar{B}} \bar{q}_{J}\left(\partial_{\bar{B}}+K_{\bar{B}}\right) \bar{X}^{J}(z) .
$$

Now we specialize to four-dimensional solutions that are supported by electric charges $Q_{I}$, magnetic charges $P^{A}$ and electric fluxes $h_{A}$. Decomposing $z^{A}=C^{A}+i \hat{X}^{A}$ and using the expression (B.13) gives

$$
\begin{aligned}
\bar{q}_{0}+\bar{q}_{A} \bar{z}^{A}= & e^{U_{4}-2 \psi-i \gamma}\left[Q_{0}+\frac{1}{2} C_{A B C} P^{A} C^{B} C^{C}+Q_{A} C^{A}-\frac{1}{2} C_{A B C} P^{A} \hat{X}^{B} \hat{X}^{C}\right. \\
& \left.+e^{2\left(\psi-U_{4}\right)} h_{A} \hat{X}^{A}+i\left(-Q_{A} \hat{X}^{A}-C_{A B C} P^{A} C^{B} \hat{X}^{C}+e^{2\left(\psi-U_{4}\right)} h_{A} C^{A}\right)\right] .
\end{aligned}
$$


Then, using (B.17) and (B.8) leads to (we recall (3.8))

$$
\begin{aligned}
\left(z^{A}\right)^{\prime}= & 2 e^{\frac{K}{2}+U_{4}-2 \psi-4 \phi} G^{A B}\left[-12 i e^{K-4 \phi} X_{B}^{5 \mathrm{~d}}\left(Q_{0}+\frac{1}{2} C_{C D E} P^{C} C^{D} C^{E}\right.\right. \\
& \left.+Q_{E} C^{E}-\frac{1}{2} C_{C D E} P^{C} X_{5 \mathrm{~d}}^{D} X_{5 \mathrm{~d}}^{E} e^{-4 \phi}+e^{2\left(\psi-U_{4}\right)} h_{E} X_{5 \mathrm{~d}}^{E} e^{-2 \phi}\right) \\
& +Q_{B}+C_{B E F} C^{E} P^{F} \\
& +12 e^{K-4 \phi} X_{B}^{5 \mathrm{~d}}\left(-Q_{E} X_{5 \mathrm{~d}}^{E} e^{-2 \phi}-C_{C D E} P^{C} C^{D} X_{5 \mathrm{~d}}^{E} e^{-2 \phi}+e^{2\left(\psi-U_{4}\right)} h_{E} C^{E}\right) \\
& \left.+i\left(e^{2\left(\psi-U_{4}\right)} h_{B}-C_{B E F} P^{E} X_{5 \mathrm{~d}}^{F} e^{-2 \phi}\right)\right] .
\end{aligned}
$$

Thus, we obtain for the real part,

$$
\begin{aligned}
\left(C^{A}\right)^{\prime}= & 2 e^{\frac{K}{2}+U_{4}-2 \psi-4 \phi} G^{A B}\left[Q_{B}+C_{B E F} C^{E} P^{F}\right. \\
& \left.+12 e^{K-4 \phi} X_{B}^{5 \mathrm{~d}}\left(-Q_{E} \hat{X}^{E}-C_{C D E} P^{C} C^{D} \hat{X}^{E}+e^{2\left(\psi-U_{4}\right)} h_{E} C^{E}\right)\right] .
\end{aligned}
$$

Next we show that the second line of this equation vanishes by virtue of the four-dimensional flow constraint

$$
\operatorname{Im}\left(e^{i \gamma} Z(Y)\right)-e^{2\left(\psi-U_{4}\right)} \operatorname{Re}\left(e^{i \gamma} W(Y)\right)=0 .
$$

We have

$$
Z(Y)=Y^{0}\left(-\frac{1}{2} C_{A B C} P^{A} z^{B} z^{C}-Q_{A} z^{A}-Q_{0}\right)
$$

and

$$
W(Y)=-Y^{0} h_{A} z^{A}
$$

This leads to

$$
\operatorname{Im}\left(e^{i \gamma} Z(Y)\right)=\left|Y^{0}\right|\left(-C_{A B C} P^{A} C^{B} \hat{X}^{C}-Q_{A} \hat{X}^{A}\right)
$$

as well as

$$
\operatorname{Re}\left(e^{i \gamma} W(Y)\right)=-\left|Y^{0}\right| h_{A} C^{A} .
$$

This gives

$$
\begin{aligned}
& \left|Y^{0}\right|\left(-C_{A B C} P^{A} C^{B} \hat{X}^{C}-Q_{A} \hat{X}^{A}+e^{2\left(\psi-U_{4}\right)} h_{A} C^{A}\right)= \\
& \quad=\operatorname{Im}\left(e^{i \gamma} Z(Y)\right)-e^{2\left(\psi-U_{4}\right)} \operatorname{Re}\left(e^{i \gamma} W(Y)\right)
\end{aligned}
$$

which vanishes due to (B.30), so that (B.29) becomes

$$
\left(C^{A}\right)^{\prime}=\frac{1}{\sqrt{2 v}} e^{V-2 B} G^{A B}\left(Q_{B}+C_{B E F} C^{E} P^{F}\right),
$$

where we used the relations (3.9) and (B.7). 
For the imaginary part of $\left(z^{A}\right)^{\prime}$ we get

$$
\begin{aligned}
\left(e^{-2 \phi} X_{5 \mathrm{~d}}^{A}\right)^{\prime}=2 e^{\frac{K}{2}+U_{4}-2 \psi-4 \phi}[ & -e^{2 \phi} X_{5 \mathrm{~d}}^{A}\left(Q_{0}+\frac{1}{2} C_{B C D} P^{B} C^{C} C^{D}+Q_{B} C^{B}\right. \\
& \left.-\frac{1}{2} C_{B C D} P^{B} X_{5 \mathrm{~d}}^{C} X_{5 \mathrm{~d}}^{D} e^{-4 \phi}+e^{2\left(\psi-U_{4}\right)} h_{B} X_{5 \mathrm{~d}}^{B} e^{-2 \phi}\right) \\
& \left.+G^{A B}\left(e^{2\left(\psi-U_{4}\right)} h_{B}-C_{B E F} P^{E} X_{5 \mathrm{~d}}^{F} e^{-2 \phi}\right)\right] \cdot
\end{aligned}
$$

Using (3.4) we obtain

$$
-C_{A B C} X_{5 \mathrm{~d}}^{C}=2 v G_{A B}-\frac{9}{v} X_{A}^{5 \mathrm{~d}} X_{B}^{5 \mathrm{~d}} .
$$

Contracting this expression once with $P^{A} X_{5 \mathrm{~d}}^{B}$ and once with $P^{A}$, we rewrite the two expressions containing $C_{B C D} P^{B} X_{5 \mathrm{~d}}^{C} X_{5 \mathrm{~d}}^{D}$ and $C_{B E F} P^{E} X_{5 \mathrm{~d}}^{F}$ in (B.37). Using (B.7) as well we obtain

$$
\begin{aligned}
\left(e^{-2 \phi} X_{5 \mathrm{~d}}^{A}\right)^{\prime}=\frac{1}{\sqrt{2 v}} e^{U_{4}-2 \psi-\phi}[ & -e^{2 \phi} X_{5 \mathrm{~d}}^{A}\left(Q_{0}+\frac{1}{2} C_{B C D} P^{B} C^{C} C^{D}+Q_{B} C^{B}\right) \\
& +2 v P^{A} e^{-2 \phi}-3 X_{5 \mathrm{~d}}^{A}\left(X_{B}^{5 \mathrm{~d}} P^{B}\right) e^{-2 \phi} \\
& \left.+e^{2\left(\psi-U_{4}\right)} G^{A B}\left(h_{B}-\frac{3}{2 v} X_{B}^{5 \mathrm{~d}}\left(h_{C} X_{5 \mathrm{~d}}^{C}\right)\right)\right] \\
=\left(e^{-2 \phi}\right)^{\prime} X_{5 \mathrm{~d}}^{A}+ & e^{-2 \phi}\left(X_{5 \mathrm{~d}}^{A}\right)^{\prime} .
\end{aligned}
$$

Using $X_{A}^{5 \mathrm{~d}}\left(X_{5 \mathrm{~d}}^{A}\right)^{\prime}=0$ (and (3.8), (3.9)) we infer

$$
\begin{gathered}
\left(e^{-2 \phi}\right)^{\prime}=\frac{1}{\sqrt{2 v}} e^{U_{4}-2 \psi-\phi}\left[-e^{2 \phi}\left(Q_{0}+\frac{1}{2} C_{B C D} P^{B} C^{C} C^{D}+Q_{B} C^{B}\right)\right. \\
\left.-e^{-2 \phi} X_{B}^{5 \mathrm{~d}} P^{B}-\frac{1}{3} e^{2\left(\psi-U_{4}\right)} h_{C} X_{5 \mathrm{~d}}^{C}\right] \\
=-\frac{1}{\sqrt{2 v}}\left[e^{-2 B-2 W+V-2 \phi}\left(Q_{0}+\frac{1}{2} C_{B C D} P^{B} C^{C} C^{D}+Q_{B} C^{B}\right)\right. \\
\left.+e^{V-2 B-2 \phi} X_{B}^{5 \mathrm{~d}} P^{B}+\frac{1}{3} e^{-U} h_{C} X_{5 \mathrm{~d}}^{C}\right]
\end{gathered}
$$

as well as

$$
\begin{aligned}
\left(X_{5 \mathrm{~d}}^{A}\right)^{\prime}=\frac{1}{\sqrt{2 v}} e^{U_{4}-2 \psi+\phi}[ & 2 e^{-2 \phi}\left(v P^{A}-X_{5 \mathrm{~d}}^{A}\left(X_{B}^{5 \mathrm{~d}} P^{B}\right)\right) \\
& \left.+e^{2\left(\psi-U_{4}\right)} G^{A B}\left(h_{B}-\frac{1}{v} X_{B}^{5 \mathrm{~d}}\left(h_{C} X_{5 \mathrm{~d}}^{C}\right)\right)\right] .
\end{aligned}
$$

The former should match the flow equation for $e^{W}$. To check this, we note that the fivedimensional flow equations (2.21) imply

$$
U^{\prime}+W^{\prime}=\frac{2}{3} h_{A} X_{5 \mathrm{~d}}^{A} e^{V}+\frac{1}{v} X_{A}^{5 \mathrm{~d}} P^{A} e^{V-2 B} .
$$


Subtracting this from the third equation of (2.21) gives

$$
W^{\prime}=\frac{1}{3} h_{A} X_{5 \mathrm{~d}}^{A} e^{V}+\frac{1}{2 v} X_{A}^{5 \mathrm{~d}} P^{A} e^{V-2 B}-\frac{1}{2} \hat{J} e^{-2 B-2 W+V},
$$

so that

$$
\left(e^{W}\right)^{\prime}=e^{-2 \phi} W^{\prime}=\frac{1}{3} h_{A} X_{5 \mathrm{~d}}^{A} e^{V-2 \phi}+\frac{1}{2 v} X_{A}^{5 \mathrm{~d}} P^{A} e^{V-2 B-2 \phi}-\frac{1}{2} \hat{J} e^{-2 B-2 W+V-2 \phi} .
$$

This matches (B.40) if we set $v=1 / 2$ and perform the identifications (3.5). Under these identifications, the flow equations (B.36) and (B.41) precisely match those for $A_{z}^{A}$ and $X_{5 \mathrm{~d}}^{A}$ appearing in (2.21). Similarly, the flow equations for the four-dimensional warp factors $U_{4}$ and $\psi$ match those of the five-dimensional warp factors $U$ and $B$ using (3.9).

\section{A different first-order rewriting}

We present a different first-order rewriting that allows for solutions with electric fields. This rewriting is the one performed in [28] for static black hole solutions, which we adapt to the case of stationary black branes in the presence of magnetic fields.

We consider the metric (2.1) and the gauge field ansatz (2.2) with $A_{z}^{A}=0$, so that $\hat{q}_{A}=q_{A}$ and $\hat{J}=J$. The starting point of the analysis is therefore Lagrangian (2.18), with $A_{z}^{A}=0, \hat{q}_{A}=q_{A}$ and $\hat{J}=J$.

We perform the following $g$-split of $U$ and $V$,

$$
\begin{aligned}
U & =U_{0}+\frac{1}{2} \log f, \\
V & =V_{0}-\frac{1}{2} \log f, \\
f & =f_{0}(r)+g^{2} f_{2}(r)=-\mu r^{2}+g^{2} e^{2 U_{2}(r)}, \\
V_{0} & =2 B(r)+W(r)+U_{0}(r)+\log (r) .
\end{aligned}
$$

In addition, we perform the rescaling

$$
P^{A}=g p^{A}, \quad J=g j,
$$

and we organize the terms in the Lagrangian into powers of $g$. This yields $\mathcal{L}=\mathcal{L}_{0}+g^{2} \mathcal{L}_{2}$. First, we analyze $\mathcal{L}_{0}$,

$$
\begin{aligned}
\mathcal{L}_{0}=e^{2 B+W+U_{0}-V_{0}} f_{0}( & 2 B^{\prime 2}+2 U^{\prime} W^{\prime}+4 B^{\prime} W^{\prime}+4 B^{\prime} U^{\prime}-G_{A B}\left(X^{A}\right)^{\prime}\left(X^{B}\right)^{\prime} \\
& \left.-\frac{1}{4} G^{A B} q_{A} q_{B} e^{-4 B-2 W+2 V_{0}}\left(f_{0}\right)^{-1}\right) .
\end{aligned}
$$

We perform a first-order rewriting of $\mathcal{L}_{0}$ by introducing parameters $\tilde{q}_{A}$ and $\gamma_{A}$ that are related to the electric charges $q_{A}$ by

$$
\frac{1}{4} G^{A B} q_{A} q_{B}=-\mu G^{A B} \tilde{q}_{A} \gamma_{B} .
$$


We obtain

$$
\begin{aligned}
\mathcal{L}_{0}= & -\mu r e^{U_{0}} \tilde{q}_{A} G^{A B}\left(6 X_{B}+e^{U_{0}}\left(r^{2} \tilde{q}_{B}-\gamma_{B}\right)\right) \\
& +e^{2 B+W+U_{0}-V_{0}} f_{0}\left[\frac{1}{2}\left(2 B^{\prime}+U_{0}^{\prime}\right)\left(2 B^{\prime}+4 W^{\prime}+3 U_{0}^{\prime}\right)\right. \\
& \left.-\frac{9}{4} G^{A B}\left(X_{A}^{\prime}-U_{0}^{\prime} X_{A}+\frac{2}{3} e^{-2 B-W+V_{0}} \tilde{q}_{A}\right)\left(X_{B}^{\prime}-U_{0}^{\prime} X_{B}+\frac{2}{3} e^{-2 B-W+V_{0}} \tilde{q}_{B}\right)\right] \\
& -2\left(e^{U_{0}} \tilde{q}_{A} X^{A} f_{0}\right)^{\prime}-\mu\left(2 W^{\prime}+4 B^{\prime}\right) .
\end{aligned}
$$

This yields the first-order flow equations

$$
\begin{aligned}
X_{A}^{\prime} & =U_{0}^{\prime} X_{A}-\frac{2}{3} e^{-2 B-W+V_{0}} \tilde{q}_{A}, \\
B^{\prime} & =-\frac{1}{2} U_{0}^{\prime}, \\
2 B^{\prime} & =-4 W^{\prime}-3 U_{0}^{\prime} .
\end{aligned}
$$

Using (C.1) as well as $X^{A}\left(X_{A}\right)^{\prime}=0$, these equations yield

$$
\begin{aligned}
W^{\prime} & =B^{\prime}=-\frac{1}{2} U_{0}^{\prime} \\
\left(e^{-U_{0}}\right)^{\prime} & =-\frac{2}{3} r X^{A} \tilde{q}_{A}, \\
\left(e^{-U_{0}} X_{A}\right)^{\prime} & =-\frac{2}{3} r \tilde{q}_{A} .
\end{aligned}
$$

Integrating the latter gives

$$
e^{-U_{0}} X_{A}=\frac{1}{3} H_{A}, \quad H_{A}=\tilde{\gamma}_{A}-r^{2} \tilde{q}_{A},
$$

where $\tilde{\gamma}_{A}$ denote integration constants. Contracting this with $X^{A}$ results in

$$
e^{-U_{0}}=\frac{1}{3} H_{A} X^{A},
$$

which satisfies the second equation of (C.7) by virtue of $X_{A}\left(X^{A}\right)^{\prime}=0$.

$\mathcal{L}_{0}$ contains, in addition, the first line, which is not the square (or the sum of squares) of a first-order flow equation. Its variation with respect to $U_{0}$ gives

$$
\tilde{q}_{A} G^{A B}\left(3 X_{B}+e^{U_{0}}\left(r^{2} \tilde{q}_{B}-\gamma_{B}\right)\right)=0 .
$$

Comparing with (C.8) yields

$$
\tilde{q}_{A} G^{A B}\left(\tilde{\gamma}_{B}-\gamma_{B}\right)=0 .
$$

Since $G^{A B}$ is positive definite, we conclude that this can only be fulfilled for arbitrary values of $\tilde{q}_{A}$ if $\tilde{\gamma}_{B}=\gamma_{B}$. On the other hand, varying the first line of $\mathcal{L}_{0}$ with respect to $X^{A}$ and using (C.8) gives

$$
-2 \mu r e^{U_{0}} \tilde{q}_{A}\left(2 \delta X^{A}+G^{A C} \delta G_{C D} X^{D}\right)
$$


which vanishes by virtue of $\delta G_{C D} X^{D}=3 \delta X_{C}=-2 G_{C D} \delta X^{D}$. Thus, we conclude that the set of variational equations derived from $\mathcal{L}_{0}$ is consistent.

Now we turn to $\mathcal{L}_{2}$,

$$
\begin{aligned}
\mathcal{L}_{2}= & e^{2 B+W+U_{0}-V_{0}+2 U_{2}}\left[-\frac{1}{2}\left(j e^{-2 B-2 W+V_{0}-U_{2}}+\left(W^{\prime}-\left(U_{0}^{\prime}+U_{2}^{\prime}\right)\right)\right)^{2}\right. \\
& -\left(B^{\prime}-\frac{1}{2}\left(U_{0}^{\prime}+U_{2}^{\prime}+W^{\prime}\right)+\frac{3}{2} X_{A} p^{A} e^{-2 B+V_{0}-U_{2}}\right)^{2} \\
& +\frac{1}{3}\left[3\left(B^{\prime}+\frac{1}{2}\left(U_{0}^{\prime}+U_{2}^{\prime}+W^{\prime}\right)\right)-2 X^{A} h_{A} e^{V_{0}-U_{2}}+\frac{3}{2} X_{A} p^{A} e^{-2 B+V_{0}-U_{2}}\right]^{2} \\
& -G_{A B}\left(X^{\prime A}-\left[\frac{2}{3} X^{C}\left(h_{C} e^{V_{0}-U_{2}}+G_{C D} p^{D} e^{-2 B+V_{0}-U_{2}}\right) X^{A}\right.\right. \\
& \left(X^{\prime B}-\left[\frac{2}{3} X^{E}\left(h_{E} e^{V_{0}-U_{2}}+h_{C} e^{V_{0}-U_{2}}+G_{C D} p^{D} e^{-2 B+V_{0}-U_{2}}\right)\right]\right) \\
& \left.\left.\left.-G^{-2 B E}\left(h_{E} e^{V_{0}-U_{2}}+G_{E F} p^{F} e^{-2 B+V_{0}-U_{2}}\right) X^{B}\right)\right]\right) \\
& +2\left(e^{2 B+W+U_{0}+U_{2}}\left(X^{A} h_{A}-\frac{3}{2} X_{A} p^{A} e^{-2 B}\right)\right)^{\prime} \\
& -\left(j e^{-W+U_{0}+U_{2}}\right)^{\prime}+2 e^{W+U_{0}+V_{0}} h_{A} p^{A}
\end{aligned}
$$

This yields the first-order flow equations

$$
\begin{aligned}
\left(X^{A}\right)^{\prime} & =\frac{2}{3} X^{C}\left(h_{C} e^{V_{0}-U_{2}}+G_{C D} p^{D} e^{-2 B+V_{0}-U_{2}}\right) X^{A}-G^{A C}\left(h_{C} e^{V_{0}-U_{2}}+G_{C D} p^{D} e^{-2 B+V_{0}-U_{2}}\right), \\
W^{\prime} & =U_{0}^{\prime}+U_{2}^{\prime}-j e^{-2 B-2 W+V_{0}-U_{2}}, \\
0 & =B^{\prime}-\frac{1}{2}\left(U_{0}^{\prime}+U_{2}^{\prime}+W^{\prime}\right)+\frac{3}{2} X_{A} p^{A} e^{-2 B+V_{0}-U_{2}}, \\
0 & =3\left(B^{\prime}+\frac{1}{2}\left(U_{0}^{\prime}+U_{2}^{\prime}+W^{\prime}\right)\right)-2 X^{A} h_{A} e^{V_{0}-U_{2}}+\frac{3}{2} X_{A} p^{A} e^{-2 B+V_{0}-U_{2}},
\end{aligned}
$$

as well as the constraint

$$
h_{A} p^{A}=0 .
$$

We have thus derived two sets of first-order flow equations (one derived from $\mathcal{L}_{0}$ and the other derived from $\mathcal{L}_{2}$ ) that need to be mutually consistent. Consistency of these two sets implies certain relations which we now derive.

Adding the third and fourth equation of (C.14) gives

$$
B^{\prime}=\frac{1}{3} X^{A} h_{A} e^{V_{0}-U_{2}}-X_{A} p^{A} e^{-2 B+V_{0}-U_{2}} .
$$

Combining this with the first equation of (C.7) yields

$$
U_{0}^{\prime}=-\frac{2}{3} X^{A} h_{A} e^{V_{0}-U_{2}}+2 X_{A} p^{A} e^{-2 B+V_{0}-U_{2}} .
$$


Comparing with the second equation of (C.7) yields

$$
\frac{1}{3} e^{-2 B-W} X^{A} \tilde{q}_{A}=-\frac{1}{3} X^{A} h_{A} e^{-U_{2}}+X_{A} p^{A} e^{-2 B-U_{2}} .
$$

Contracting the first equation of (C.14) with $G_{A B}$ results in

$$
X_{A}^{\prime}=-\left(\frac{2}{3} X^{C} h_{C} e^{V_{0}-U_{2}}+X_{C} p^{C} e^{-2 B+V_{0}-U_{2}}\right) X_{A}+\frac{2}{3}\left(h_{A} e^{V_{0}-U_{2}}+G_{A D} p^{D} e^{-2 B+V_{0}-U_{2}}\right),
$$

Using (C.17) gives

$$
X_{A}^{\prime}=\left(U_{0}^{\prime}-3 X_{C} p^{C} e^{-2 B+V_{0}-U_{2}}\right) X_{A}+\frac{2}{3}\left(h_{A} e^{V_{0}-U_{2}}+G_{A D} p^{D} e^{-2 B+V_{0}-U_{2}}\right) .
$$

Using $W^{\prime}=-\frac{1}{2} U_{0}^{\prime}$ (from the first equation of (C.7)) in the second equation of (C.14) gives

$$
U_{2}^{\prime}=-\frac{3}{2} U_{0}^{\prime}+j e^{-2 B-2 W+V_{0}-U_{2}},
$$

which expresses $U_{2}$ in terms of $U_{0}$.

The second equation of (C.14) can be rewritten as

$$
U_{0}^{\prime}+U_{2}^{\prime}+W^{\prime}=2 W^{\prime}+j e^{-2 B-2 W+V_{0}-U_{2}}
$$

which, when inserted into the third equation of (C.14), gives

$$
-j e^{-2 W}+3 X_{A} p^{A}=0 .
$$

Using $-2 W=U_{0}$ (ignoring an additive constant) as well as (C.8), this results in

$$
j=H_{A} p^{A} .
$$

Since the left hand side is constant, we conclude that

$$
\tilde{q}_{A} p^{A}=0, \quad j=\gamma_{A} p^{A} .
$$

Finally, comparing the first equation of (C.6) with (C.20)

$$
-3 X_{C} p^{C} e^{-2 B-U_{2}} X_{A}+\frac{2}{3}\left(h_{A} e^{-U_{2}}+G_{A D} p^{D} e^{-2 B-U_{2}}\right)+\frac{2}{3} \tilde{q}_{A} e^{-2 B-W}=0 .
$$

Note that the contraction of (C.26) with $X^{A}$ gives back (C.18). On the other hand, contracting (C.26) with $p^{A}$ and using (C.15) and (C.25) gives

$$
3\left(X_{A} p^{A}\right)^{2}=\frac{2}{3} p^{A} G_{A B} p^{B} .
$$

Observe that both sides are positive definite. This relation is, for instance, satisfied for the STU-model $X^{1} X^{2} X^{3}=1$. Inserting the relation

$$
G_{A B}=-\frac{1}{2} C_{A B C} X^{C}+\frac{9}{2} X_{A} X_{B}
$$


into (C.27) we obtain

$$
X^{A} C_{A B C} p^{B} p^{C}=0 .
$$

Thus, we conclude that the two sets (C.7) and (C.14) are mutually consistent, provided that (C.26) is satisfied and the constraints (C.15) and (C.25) hold.

In the following, we solve the first-order flow equations for the case when $p^{A}=0$. Then $j=0$, so that from (C.21) we obtain $U_{2}^{\prime}=-\frac{3}{2} U_{0}^{\prime}$, which also equals $W^{\prime}+2 B^{\prime}$ by virtue of the first equation of (C.7). Thus $U_{2}=W+2 B$, up to an additive constant. Then, (C.26) is satisfied provided we set $\tilde{q}_{A}=-h_{A}$. Summarizing, when $p^{A}=0$, we obtain

$$
\begin{aligned}
\frac{1}{4} G^{A B} q_{A} q_{B} & =\mu G^{A B} h_{A} \gamma_{B}, \\
e^{-U_{0}} X_{A} & =\frac{1}{3} H_{A}, \quad H_{A}=\gamma_{A}+r^{2} h_{A}, \\
e^{-U_{0}} & =\frac{1}{3} H_{A} X^{A}, \\
B & =W=-\frac{1}{2} U_{0}, \\
U_{2} & =-\frac{3}{2} U_{0}, \\
V_{0} & =-\frac{1}{2} U_{0}+\log r, \\
f & =-\mu r^{2}+g^{2} e^{-3 U_{0}}, \\
\left(e^{A}\right)^{\prime} & =\frac{1}{2} e^{2 U_{0}} r G^{A B} q_{B} .
\end{aligned}
$$

This is the black brane analog of the black hole solutions discussed in [28].

For this class of solutions, we now check the Hamiltonian constraint

$$
R_{t t}+\frac{\delta \mathcal{L}_{M}}{\delta g^{t t}}-\frac{1}{2} g_{t t}\left(R+\mathcal{L}_{M}\right)=0
$$

where $\mathcal{L}_{M}$ denotes the matter Lagrangian. Using

$$
\sqrt{-g} g^{t t}\left(R_{t t}-\frac{1}{2} g_{t t} R\right)=-3 e^{3 B+U-V}\left(B^{\prime 2}+B^{\prime} U^{\prime}\right)+3\left(e^{3 B+U-V} B^{\prime}\right)^{\prime},
$$

as well as

$$
\begin{aligned}
& \frac{\delta \mathcal{L}_{M}}{\delta g^{t t}}-\frac{1}{2} g_{t t} \mathcal{L}_{M} \\
& \quad=\frac{1}{2} g_{t t}\left[e^{-6 B} G^{A B} q_{A} q_{B}+e^{-2 V} G_{A B} X^{\prime A} X^{B}+g^{2}\left(G^{A B}-2 X^{A} X^{B}\right) h_{A} h_{B}\right],
\end{aligned}
$$

where we replaced the electric fields by their charges, we obtain for (C.31),

$$
\mathcal{L}_{0}+\mathcal{L}_{2}-6\left(e^{3 B+U-V} B^{\prime}\right)^{\prime}=0 .
$$

Imposing the first-order flow equations, this reduces to

$$
\begin{aligned}
& \left(e^{U_{0}} f_{0} \tilde{q}_{A} X^{A}+3 \mu B\right)^{\prime}+g^{2}\left(e^{3 B+U_{0}+U_{2}} \tilde{q}_{A} X^{A}\right)^{\prime}+3\left(e^{3 B+U_{0}-V_{0}} f B^{\prime}\right)^{\prime} \\
& +\mu r e^{U_{0}} \tilde{q}_{A} X^{A}=0 .
\end{aligned}
$$


Then, using the second equation of (C.7) and

$$
3 r B^{\prime \prime}=-U_{0}^{\prime} e^{U_{0}} r^{2} \tilde{q}_{A} X^{A}-e^{U_{0}} r \tilde{q}_{A} X^{A}-e^{U_{0}} r^{2} \tilde{q}_{A} X^{\prime A},
$$

we find that (C.35) is satisfied on a solution to the first-order flow equations. Thus, the Hamiltonian constraint (C.31) does not lead to any further constraint.

The class of static solutions (C.30) was obtained long time ago in [27] by solving the equations of motion. Their mass density is determined by $\mu$. Let us consider a black solution, with the horizon located at $f\left(r_{H}\right)=0$ with $e^{2 U_{0}}\left(r_{H}\right) \neq 0$. Its temperature is then given by

$$
T_{H}=\left[\frac{e^{U_{0}-V_{0}}}{4 \pi}\left|f^{\prime}\right|\right]_{r=r_{H}}=\left[\frac{e^{\frac{3}{2} U_{0}}}{4 \pi r}\left|f^{\prime}\right|\right]_{r=r_{H}},
$$

where we used (C.30) to express $V_{0}$ in terms of $U_{0}$. The horizon condition $f\left(r_{H}\right)=0$ gives

$$
\mu r_{H}^{2}=g^{2} e^{-3 U_{0}\left(r_{H}\right)}
$$

and hence $r_{H} \neq 0$. For the solution to be extremal, its temperature has to vanish. Imposing $f^{\prime}\left(r_{H}\right)=0$ results in

$$
\mu r_{H}+\frac{3}{2} g^{2} e^{-3 U_{0}\left(r_{H}\right)} U_{0}^{\prime}\left(r_{H}\right)=0 .
$$

Combining both equations gives

$$
1+\frac{3}{2} r_{H} U_{0}^{\prime}\left(r_{H}\right)=0
$$

As an example, consider the STU-model $X^{1} X^{2} X^{3}=1$ and take $\gamma_{A}=c h_{A}$ with $c>0$, so that $H_{A}=h_{A} H$ with $H=c+r^{2}$. Then

$$
\begin{array}{rlrl}
e^{-3 U_{0}} & =H_{1} H_{2} H_{3}=h_{1} h_{2} h_{3} H^{3}, & X^{1}=\frac{\left(H_{2} H_{3}\right)^{1 / 3}}{H_{1}^{2 / 3}}=\frac{\left(h_{2} h_{3}\right)^{1 / 3}}{h_{1}^{2 / 3}}, \\
X^{2}=\frac{\left(H_{1} H_{3}\right)^{1 / 3}}{H_{2}^{2 / 3}}=\frac{\left(h_{1} h_{3}\right)^{1 / 3}}{h_{2}^{2 / 3}}, & X^{3}=\frac{\left(H_{1} H_{2}\right)^{1 / 3}}{H_{3}^{2 / 3}}=\frac{\left(h_{1} h_{2}\right)^{1 / 3}}{h_{3}^{2 / 3}} .
\end{array}
$$

The scalars $X^{A}$ are thus constant. We set $g=1$ and $\alpha^{3}=h_{1} h_{2} h_{3}>0$. The horizon condition (C.38) yields

$$
\mu H\left(r_{H}\right)-\alpha^{3} H^{3}\left(r_{H}\right)=\mu c
$$

while the condition (C.39) gives

$$
r_{H}\left(\mu-3 \alpha^{3} H^{2}\left(r_{H}\right)\right)=0,
$$

which implies (we take $r_{H} \neq 0$ ),

$$
\mu=3 \alpha^{3} H^{2}\left(r_{H}\right)
$$

Inserting (C.44) into (C.42) gives

$$
2 \alpha^{3} H^{3}\left(r_{H}\right)=\mu c .
$$


Combining (C.45) with the first equation of (C.30), which takes the form

$$
\mu c=\frac{1}{4} \frac{q_{A} G^{A B} q_{B}}{h_{A} G^{A B} h_{B}},
$$

yields the entropy density as

$$
\mathcal{S}=\frac{1}{4} e^{3 B\left(r_{H}\right)}=\frac{1}{4}\left(\alpha^{3} H^{3}\left(r_{H}\right)\right)^{1 / 2}=\frac{1}{4}\left(\frac{\mu c}{2}\right)^{1 / 2}=\frac{1}{8}\left(\frac{1}{2} \frac{q_{A} G^{A B} q_{B}}{h_{A} G^{A B} h_{B}}\right)^{1 / 2} .
$$

Extremal electric solutions of this type have been considered recently in [7, 24].

Open Access. This article is distributed under the terms of the Creative Commons Attribution License which permits any use, distribution and reproduction in any medium, provided the original author(s) and source are credited.

\section{References}

[1] J.M. Maldacena, The large- $N$ limit of superconformal field theories and supergravity, Adv. Theor. Math. Phys. 2 (1998) 231 [Int. J. Theor. Phys. 38 (1999) 1113] [hep-th/9711200] [INSPIRE].

[2] S.L. Cacciatori and D. Klemm, Supersymmetric AdS 4 black holes and attractors, JHEP 01 (2010) 085 [arXiv:0911.4926] [inSPIRE].

[3] G. Dall'Agata and A. Gnecchi, Flow equations and attractors for black holes in $N=2 \mathrm{U}(1)$ gauged supergravity, JHEP 03 (2011) 037 [arXiv: 1012.3756] [INSPIRE].

[4] K. Hristov and S. Vandoren, Static supersymmetric black holes in AdS $S_{4}$ with spherical symmetry, JHEP 04 (2011) 047 [arXiv: 1012.4314] [INSPIRE].

[5] S. Kachru, R. Kallosh and M. Shmakova, Generalized Attractor Points in Gauged Supergravity, Phys. Rev. D 84 (2011) 046003 [arXiv: 1104.2884] [InSPIRE].

[6] S. Barisch, G.L. Cardoso, M. Haack, S. Nampuri and N.A. Obers, Nernst branes in gauged supergravity, JHEP 11 (2011) 090 [arXiv:1108.0296] [INSPIRE].

[7] A. Donos, J.P. Gauntlett and C. Pantelidou, Magnetic and Electric AdS Solutions in Stringand M-theory, Class. Quant. Grav. 29 (2012) 194006 [arXiv:1112.4195] [inSPIRE].

[8] P. Meessen and T. Ortín, Supersymmetric solutions to gauged $N=2 D=4$ SUGRA: the full timelike shebang, Nucl. Phys. B 863 (2012) 65 [arXiv: 1204.0493] [INSPIRE].

[9] K. Goldstein, S. Kachru, S. Prakash and S.P. Trivedi, Holography of charged dilaton black holes, JHEP 08 (2010) 078 [arXiv:0911.3586] [InSPIRE].

[10] K. Goldstein, N. Iizuka, S. Kachru, S. Prakash, S.P. Trivedi, et al., Holography of Dyonic Dilaton Black Branes, JHEP 10 (2010) 027 [arXiv:1007.2490] [INSPIRE].

[11] P. Berglund, J. Bhattacharyya and D. Mattingly, Charged dilatonic AdS black branes in arbitrary dimensions, JHEP 08 (2012) 042 [arXiv:1107.3096] [INSPIRE].

[12] E. D'Hoker and P. Kraus, Magnetic Field Induced Quantum Criticality via new Asymptotically Ad $S_{5}$ Solutions, Class. Quant. Grav. 27 (2010) 215022 [arXiv:1006.2573] [INSPIRE].

[13] T. Faulkner, G.T. Horowitz and M.M. Roberts, Holographic quantum criticality from multi-trace deformations, JHEP 04 (2011) 051 [arXiv: 1008.1581] [INSPIRE]. 
[14] J. Erdmenger, V. Grass, P. Kerner and T.H. Ngo, Holographic superfluidity in imbalanced mixtures, JHEP 08 (2011) 037 [arXiv:1103.4145] [INSPIRE].

[15] E. D'Hoker and P. Kraus, Quantum criticality via magnetic branes, arXiv:1208.1925 [INSPIRE].

[16] A. Chamblin, R. Emparan, C.V. Johnson and R.C. Myers, Charged AdS black holes and catastrophic holography, Phys. Rev. D 60 (1999) 064018 [hep-th/9902170] [INSPIRE].

[17] J.B. Gutowski and H.S. Reall, Supersymmetric AdS $S_{5}$ black holes, JHEP 02 (2004) 006 [hep-th/0401042] [INSPIRE].

[18] H.K. Kunduri and J. Lucietti, Near-horizon geometries of supersymmetric AdS $S_{5}$ black holes, JHEP 12 (2007) 015 [arXiv:0708.3695] [INSPIRE].

[19] J. Grover, J.B. Gutowski and W. Sabra Null half-supersymmetric solutions in five-dimensional supergravity, JHEP 10 (2008) 103 [arXiv:0802.0231] [INSPIRE].

[20] E. D'Hoker and P. Kraus, Magnetic brane solutions in AdS, JHEP 10 (2009) 088 [arXiv:0908.3875] [INSPIRE].

[21] E. D'Hoker and P. Kraus, Charged Magnetic Brane Solutions in AdS $S_{5}$ and the fate of the third law of thermodynamics, JHEP 03 (2010) 095 [arXiv:0911.4518] [INSPIRE].

[22] A. Almuhairi, $A d S_{3}$ and $A d S_{2}$ Magnetic Brane Solutions, arXiv:1011.1266 [INSPIRE].

[23] A. Almuhairi and J. Polchinski, Magnetic AdS $\times R^{2}$ : Supersymmetry and stability, arXiv:1108.1213 [INSPIRE].

[24] A. Donos and J.P. Gauntlett, Holographic helical superconductors, JHEP 12 (2011) 091 [arXiv:1109.3866] [INSPIRE].

[25] S. Bellucci, S. Ferrara, A. Shcherbakov and A. Yeranyan, Attractors and first order formalism in five dimensions revisited, Phys. Rev. D 83 (2011) 065003 [arXiv: 1010.3516] [InSPIRE].

[26] J. de Boer, M.C. Cheng, R. Dijkgraaf, J. Manschot and E. Verlinde, A Farey tail for attractor black holes, JHEP 11 (2006) 024 [hep-th/0608059] [INSPIRE].

[27] K. Behrndt, M. Cvetič and W. Sabra, Nonextreme black holes of five-dimensional $N=2$ AdS supergravity, Nucl. Phys. B 553 (1999) 317 [hep-th/9810227] [INSPIRE].

[28] G.L. Cardoso and V. Grass, On five-dimensional non-extremal charged black holes and FRW cosmology, Nucl. Phys. B 803 (2008) 209 [arXiv: 0803.2819] [INSPIRE].

[29] M. Günaydin, G. Sierra and P. Townsend, Vanishing potentials in gauged $N=2$ supergravity: an application of Jordan algebras, Phys. Lett. B 144 (1984) 41 [INSPIRE].

[30] M. Günaydin, G. Sierra and P. Townsend, Gauging the D = 5 Maxwell-Einstein Supergravity Theories: More on Jordan Algebras, Nucl. Phys. B 253 (1985) 573 [InSPIRE].

[31] G.L. Cardoso, J.M. Oberreuter and J. Perz, Entropy function for rotating extremal black holes in very special geometry, JHEP 05 (2007) 025 [hep-th/0701176] [INSPIRE].

[32] M. Bañados, C. Teitelboim and J. Zanelli, The black hole in three-dimensional space-time, Phys. Rev. Lett. 69 (1992) 1849 [hep-th/9204099] [INSPIRE].

[33] P. Kraus, Lectures on black holes and the AdS $S_{3} / C F T_{2}$ correspondence, Lect. Notes Phys. 755 (2008) 193 [hep-th/0609074] [INSPIRE].

[34] S. Harrison, S. Kachru and H. Wang, Resolving Lifshitz horizons, arXiv:1202.6635 [INSPIRE].

[35] B. de Wit, $N=2$ symplectic reparametrizations in a chiral background, Fortsch. Phys. 44 (1996) 529 [hep-th/9603191] [INSPIRE]. 